The Astrophysical Journal Supplement Series, 176:164-183, 2008 May

(C) 2008. The American Astronomical Society. All rights reserved. Printed in U.S.A.

\title{
THE SPECTRAL ENERGY DISTRIBUTION OF SELF-GRAVITATING INTERSTELLAR CLOUDS. I. SPHERES
}

\author{
Jörg Fischera ${ }^{1}$ and Michael A. Dopita \\ Research School of Astronomy \& Astrophysics, Institute of Advanced Studies, Australian National University, \\ Cotter Road, Weston Creek, ACT 2611, Australia; fischera@cita.utoronto.ca, mad@mso.anu.edu.au \\ Received 2006 April 7; accepted 2007 November 2
}

\begin{abstract}
We derive the spectral energy distribution (SED) of dusty, isothermal, self-gravitating, stable and spherical clouds externally heated by the ambient interstellar radiation field (ISRF). For a given radiation field and dust properties, the radiative transfer problem is determined by the pressure of the surrounding medium and by the cloud mass expressed as a fraction of the maximum stable cloud mass above which the clouds become gravitationally unstable. To solve the radiative transfer problem, a ray-tracing code is used to accurately derive the light distribution inside the cloud. This code considers both anisotropic scattering on dust grains and multiple scattering events. The dust properties inside the clouds are assumed to be the same as in the diffuse interstellar medium in our Galaxy. We analyze the effect of the pressure, the critical mass fraction, and the ISRF on the SED and present brightness profiles in the visible, the IR/FIR, and the submillimeter/millimeter regime with the focus on the scattered emission and the thermal emission from PAH molecules and dust grains.
\end{abstract}

Subject headings: dust, extinction - ISM: clouds — radiative transfer

\section{INTRODUCTION}

Self-gravitating clouds are an essential constituent of the inhomogeneous interstellar medium (ISM). They are either regions of ongoing or, if the clouds become cold enough to be gravitationally unstable, future star formation. They are important for the chemistry in the ISM through the formation of molecules, in particular, molecular hydrogen, and they probably play an essential role for the life cycle of interstellar dust grains since in dense environments grains are thought to grow to larger sizes through a variety of physical processes. Those grain processes not only alter the mean optical grain properties and therefore the extinction curve, most importantly at ultraviolet (UV) and far-ultraviolet (FUV) wavelengths, but will probably also lead to a different integrated grain surface area. Because molecules form on dust grains and their formation rate is controlled by the strength of the photodissociation flux inside the cloud, these grain processes will have direct consequences for the formation of molecules. Our knowledge of the dust properties in clouds is furthermore crucial for our understanding of the origin of the interstellar extinction curve as parts of the dense material will be redistributed into the diffuse ISM. This will enrich the gas with large grains with a composition possibly rather different to grains initially formed around cold stars, in planetary nebulae, or in the metalrich part of the ejected material of supernova explosions.

The spectral energy distribution (SED) of self-gravitating clouds might have an important contribution to the global SED of galaxies, in particular, at far-infrared (FIR) and submillimeter wavelengths, thanks to the large population of cool dust that they are expected to contain.

The origin of self-gravitating clouds is likely related to turbulent motion. A turbulent medium would not only naturally produce a distribution of cloud masses as is observed for the ISM (Elmegreen 2002) but is also possibly directly linked to the initial mass function of the stars (Padoan et al.1997; Padoan \& Nordlund 2002; Bate et al. 2003). An isothermal turbulent me-

\footnotetext{
${ }^{1}$ Current address: CITA, University of Toronto, 60 St. George Street, Toronto, ON M5S 3H8, Canada.
}

dium leads to a multifractal structure where large clouds actually consist of distributions of clouds of different sizes. As shown by simulations ( Vázquez-Semadeni 1994; Padoan et al. 1997; Passot \& Vázquez-Semadeni 1998) and verified analytically (Nordlund \& Padoan 1999), the probability distribution of the local density in an isothermal turbulent medium can be well described by a simple lognormal density distribution. In the presence of gravity this distribution is squeezed by increasing the number of higher densities while the number of low-density values decreases (Nordlund \& Padoan 1999). This happens as clouds become more compact due to gravitational pressure. If the gravitational forces are strong enough to hold the cloud together against the turbulent motions, the density enhancements become stable. If the gravitational pressure surpasses the maximum thermal and, possibly, magnetic pressure support, then they will collapse and will form stars.

Here, we consider stable, isothermal, self-gravitating clouds assumed to be in pressure equilibrium with their surrounding medium. To be able to infer the dust properties from observations of the reemitted and the scattered light and to minimize any complications by radiative transfer effects, we limit our attention to simple, idealized, spherical cloud structures. We find them in small molecular clouds, the so-called Bok globules (Bok \& Reilly 1947), and, to a somewhat lesser extent, in translucent clouds, so named as they have attenuation in the range $1<A_{V}<5$ (van Dishoeck $\&$ Black 1988). We furthermore consider only those isolated clouds that are illuminated by the interstellar radiation field (ISRF), which is, to first approximation, assumed to be isotropic.

Clouds often show a rather elongated structure that might be idealized through self-gravitating infinite cylinders. The SED of those clouds is analyzed in a following paper (Paper II in this series).

Translucent clouds are the interface between the diffuse and the dense molecular phase of the cold neutral medium (CNM). Although the clouds are optically thick, they are still transparent enough to allow optical and UV absorption line measurements that enable us to analyze the different processes in dense gas in more detail. Measurements of the extinction curve, for example (Cardelli \& Savage 1988; Boulanger et al. 1994; Rachford et al. 
2002), give us essential information about the grain properties in clouds that is important for inferring the conditions and the processes responsible for grain growth.

Bok globules are highly optically thick at their centers and appear as dark clouds in front of distant stars and nebulae. These clouds are through their simple, almost spherical shape ideal objects for studying the formation of low massive stars and the molecular phase of the ISM. That those clouds are indeed regions of star formation as suggested by Bok \& Reilly (1947) has been revealed by an Infrared Astronomical Satellite (IRAS) survey that showed that $23 \%$ of Bok globules are associated with a young stellar object with a mass of at least $0.7 M_{\odot}$, the detection limit of the survey, in the cloud center (Yun \& Clemens 1990).

The scattered light from almost spherical globules that are illuminated by an idealized isotropic radiation field has been used not only to infer the properties of the grains such as the typical size, the dust albedo, and the asymmetry of the scattered light but also to derive the density profile at the rim of the cloud (Mattila 1970; Fitzgerald et al. 1976; Witt et al. 1990; Lehtinen \& Mattila 1996).

Molecular line observations show that Bok globules have, in general, at their outskirts a rather steep density profile approximately described by a power law $n(r) \propto r^{-2}$ (Arquilla \& Goldsmith 1985). In the center of stable clouds the profile becomes rather flat (Dickmann \& Clemens 1983; Lehtinen et al. 1995). Qualitatively the density profile of translucent clouds is similar but shallower at the outskirts.

The questions we seek to address in this paper are what is the SED of stable self-gravitating clouds and how is the scattered light and the thermal reemission affected by the density structure of these clouds? Although there are several indications that the grain properties in dense clouds are different than in the diffuse ISM, we base our calculations on the mean properties derived for the Milky Way. We address the effect of different grain properties in $\S 6$.

Calculations of the SED from isothermal self-gravitating clouds were presented by Evans et al. (2001) and Stamatellos \& Whitworth (2003), but they used a different approach to model the dust emission. We discuss their models in more detail below.

Our work may be best compared with that presented by Bernard et al. (1992). The model assumptions, however, are different in several respects. In particular it was not their intention to model the scattered light as it is here. Furthermore, the calculation in this paper is based on a physical, although simple, cloud model, while the results by Bernard et al. were based on a simplified density structure with a flat density profile in the cloud center and a power-law profile in the outer cloud region $n(r) \propto r^{-\beta}$ with $\beta$ chosen to be 0,1 , and 2 . For a fixed density at the cloud boundary, their SEDs were derived for several values of the central density, the power $\beta$, and the total extinction $A_{V}$ through the cloud center. In our model of stable clouds the SEDs are determined by only two parameters, the pressure outside of the cloud and the critical mass fraction, which is the cloud mass relative to the critical mass for which the cloud becomes unstable against gravitational collapse. The calculations of Bernard et al. and ourselves are based on the mean properties of the dust grains in the ISM.

The paper is outlined as follows: In $\S 2$ we discuss the physical properties of the isothermal cloud model. In $\S 3$ we describe the dust model used in all calculations and in $\S 4$ the radiative transfer program. In $\S 5$ we adopt this model to derive the SED and brightness profiles for a number of different parameters. We show the effect of the compactness of the clouds on the emission of polycyclic aromatic hydrocarbon (PAH) molecules and the dust emission. The results are discussed in $\S 6$. A summary of our results is given in $\S 7$.

\section{MODEL OF ISOTHERMAL CLOUDS}

To model the SED of self-gravitating clouds, we consider the clouds to be isothermal and to be in pressure equilibrium with the surrounding medium. Particular interest is given for clouds embedded in the warm neutral medium (WNM) of our Galaxy. The pressure of this medium, which includes the thermal and magnetic pressure, is taken to be $p_{\text {ext }} / k=2 \times 10^{4} \mathrm{~K} \mathrm{~cm}^{-3}$ based on the work from Boulares \& Cox (1990). Following Curry \& McKee (2000), the pressure due to cosmic rays is neglected as they will pervade both the cloud and the ambient medium. For simplicity the isothermal clouds are taken to be spherical. In addition we neglect the observed complex substructure likely related to turbulent motion inside the clouds. The clouds have a radial density profile that is determined by the equilibrium of the isothermal and gravitational pressure, which is obtained by solving the LaneEmden equation. The mathematical structure of such clouds is developed in the Appendix. We point out that our approach stays valid in the case where the turbulent motion and magnetic pressure inside the cloud can be approximated by an increase of the thermal motion.

The problem of isothermal clouds plays an important role in astrophysics as it gives insights into the inner structure of stars and their formation and therefore had been studied in the past (Kippenhahn \& Weigert 1990). Isothermal spheres have furthermore been used to analyze extinction measurements (Alves et al. 2001; Kandori et al. 2005) and to model the observed IR emission of dense clouds (Evans et al. 2001) often referred to as prestellar cores. In general very good agreement has been found. In the following we describe some properties that are important for the solution of the radiative transfer problem.

\subsection{The Critical Cloud Mass}

It is well known from the application of the Lane-Emden equation that the clouds can only remain stable against gravitational collapse for masses below a critical value, $M_{\mathrm{cl}, \max }$. This critical mass is given by

$$
\begin{aligned}
\left(\frac{M_{\mathrm{cl}, \max }}{M_{\odot}}\right)= & 2029\left(\frac{T_{\mathrm{cl}}}{100 \mathrm{~K}}\right)^{2} \\
& \times\left(\frac{\mu}{1.0}\right)^{-2}\left(\frac{p_{\mathrm{ext}} / k}{10^{4} \mathrm{~K} \mathrm{~cm}^{-3}}\right)^{-1 / 2} .
\end{aligned}
$$

\subsection{The Density Profile}

The density profile of isothermal clouds depends on the critical mass fraction $f=M_{\mathrm{cl}} / M_{\mathrm{cl} \text {, max }}$ of the clouds' mass $M_{\mathrm{cl}}$ and the maximum stable mass $M_{\mathrm{cl} \text {, max }}$. In general the profile becomes steeper for higher mass fraction $f$ (Fig. 1). Close to the cloud center the profile flattens. Apart from clouds close to the maximum cloud mass, the density profile outside the central region is shallower than a power law $\rho \propto r^{-2}$.

For a given mass fraction the density increases proportionally to $p_{\text {ext }} \mu / T_{\mathrm{cl}}$. The relationship between the central density and mass fraction $f$, outer pressure $p$, and cloud temperature $T$ is shown in Figure 2, where the mean molecular weight is taken to be 2.36, consistent with a gas with solar abundance where hydrogen is completely molecular.

A self-gravitating isothermal cloud at the limit of its critical mass against gravitational collapse is known as a Bonnor-Ebert sphere (Ebert 1955; Bonnor 1956). This is characterized by an overpressure of 14.04 in the center relative to the outer medium. As the central density is very sensitive close to the critical mass 


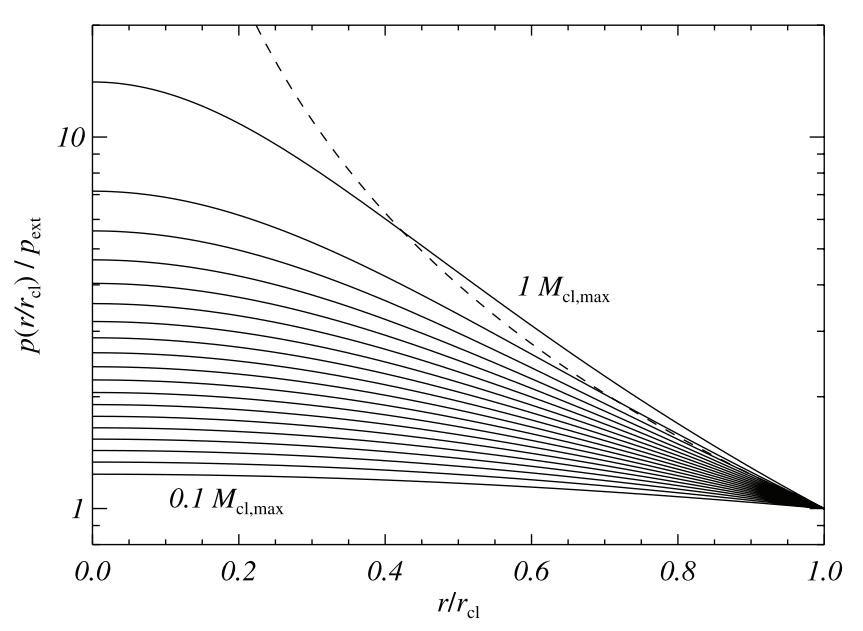

FIG. 1.-Pressure (or density) profile $p\left(r / r_{\mathrm{cl}}\right) / p_{\text {ext }}$ of isothermal clouds. The cloud mass is given as a fraction of the maximum cloud mass $M_{\mathrm{cl} \text {,max }}$ of noncollapsing clouds and is varied from 0.1 to 1 in steps of 0.05 . For comparison a density profile $n(r) \propto r^{-2}$ is also shown (dashed line).

and decreases strongly toward slightly smaller masses, stable clouds should be characterized with overpressures of less than a factor of 10 . A cloud with a mass fraction of $f=0.9$ has, for example, an overpressure of less than 6.

\subsection{The Cloud Size}

The cloud size is not a critical value for the radiative transport problem as this is entirely determined (for given dust properties and an external radiation field) by the critical mass fraction $f=$ $M_{\mathrm{cl}} / M_{\mathrm{cl} \text {, max }}$ and the outer pressure $p_{\text {ext }}$. However, the size determines the total cloud luminosity and is important from an observational point of view. For a given critical mass fraction the cloud radius is proportional to $T_{\mathrm{cl}} \mu^{-1} p_{\mathrm{ext}}^{-1 / 2}$. A cloud that is 10 times as hot is also 10 times larger, but it has 100 times more mass. In Figure 3 the cloud radius is compared with a simplified model of homogeneous spheres as a function of the critical mass fraction $f$. The two curves start to deviate for critical mass fractions larger than $\sim 0.1$, where the density profile becomes significantly steeper with increasing $f$. As more mass is concentrated in the cloud center, the cloud size becomes smaller with respect to the homo-

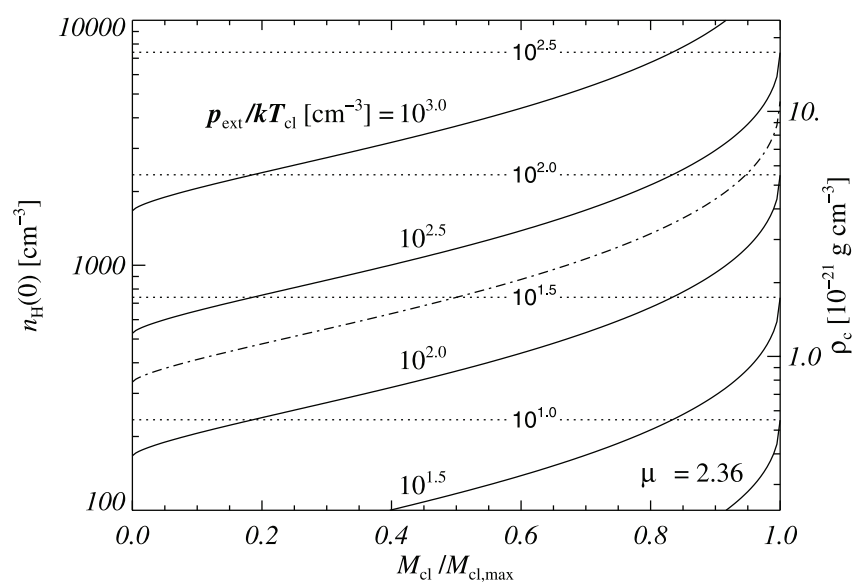

FIG. 2.-Central density in isothermal clouds as a function of the critical mass fraction $f$ for several values of $p_{\text {ext }} / k T_{\mathrm{cl}}$. The dash-dotted curve shows the density values for the parameters assumed in the paper. The maximum central densities for different parameters $p_{\text {ext }} / k T_{\mathrm{cl}}$ are shown as dotted horizontal lines. The abundances are taken to be solar, and the hydrogen is assumed to be completely in the form of molecular hydrogen, giving a mean molecular weight $\mu \sim 2.36$.

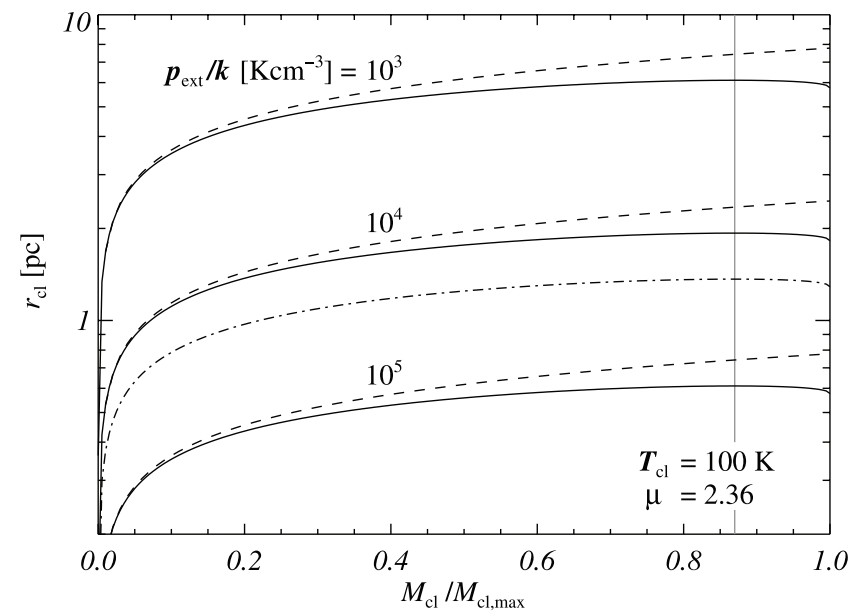

FIG. 3.-Cloud size as a function of the critical mass fraction $f=M_{\mathrm{cl}} / M_{\mathrm{cl} \text {, } \max }$ for various values of the external pressure $p_{\text {ext }} / k$ (solid lines). The cloud temperature is taken to be $100 \mathrm{~K}$, and the mean molecular weight is taken to be $\mu=2.36$. The dash-dotted curve shows the corresponding cloud sizes for our model assumption $\left(p_{\text {ext }} / k=2 \times 10^{4} \mathrm{~K} \mathrm{~cm}^{-3}\right)$. For comparison the sizes of homogeneous filled clouds are also shown (dashed lines). The vertical line marks the maximum cloud extension for $f=0.8698$.

geneous sphere. Above $f=0.8698$ the cloud size shrinks with increasing critical mass fraction.

\subsection{The Column Density $N_{\mathrm{H}}$}

The most important parameter that determines the radiative transfer problem is the column density toward the cloud center. For a given outer pressure $p_{\text {ext }} / k$ the column density $N_{\mathrm{H}}$ of all hydrogen depends only on the ratio $f=M_{\mathrm{cl}} / M_{\mathrm{cl} \text {, max }}$. For a given mass fraction $f$ the column density increases proportionally to the square root of the outer pressure:

$$
N_{\mathrm{H}}\left(f, p_{\text {ext }}\right)=\sqrt{\frac{p_{\text {ext }}}{p_{\text {ext }}^{\prime}}} N_{\mathrm{H}}\left(f, p_{\text {ext }}^{\prime}\right) .
$$

However, the critical mass decreases with pressure. Therefore, in higher pressure regions the clouds are less massive but more compact and more opaque.

The variation of column density as a function of the critical mass fraction $f$ is shown in Figure 4. This is again compared with the column density through an homogeneous sphere. For small critical mass fractions both values are the same. Toward higher critical mass fractions the column density through the isothermal clouds increases strongly relative to the homogeneous sphere. For stable isothermal clouds embedded in the WNM with $p_{\text {ext }} / k=2 \times 10^{4} \mathrm{~K} \mathrm{~cm}^{-3}$, one expects total column densities no larger than $N_{\mathrm{H}}=1.6 \times 10^{22} \mathrm{~cm}^{-2}$, a value slightly smaller than the column density through molecular clouds $\left(N_{\mathrm{H}}=2 N_{\mathrm{H}_{2}} \sim 2 \times\right.$ $10^{22} \mathrm{~cm}^{-2}$; Dopita \& Sutherland 2003). Gravitationally stable clouds should have total column densities $N_{\mathrm{H}}$ of a few times $10^{21} \mathrm{~cm}^{-2}$, a value that is quite similar to the column densities for translucent clouds (van Dishoeck \& Black 1988; Rachford et al. 2002).

In Figure 4 we also give the attenuation toward the cloud center in the visual, assuming typical dust properties. Clouds with column densities of $10^{21} \mathrm{~cm}^{-2}$ have an attenuation of $A_{V} \approx 0.5$. The attenuation toward the center of clouds in the WNM of our Galaxy is not larger than 4. Typical clouds in the WNM are therefore marginally optically thick in the optical. Self-gravitating clouds with high critical mass fraction fulfill the criterion of translucent 


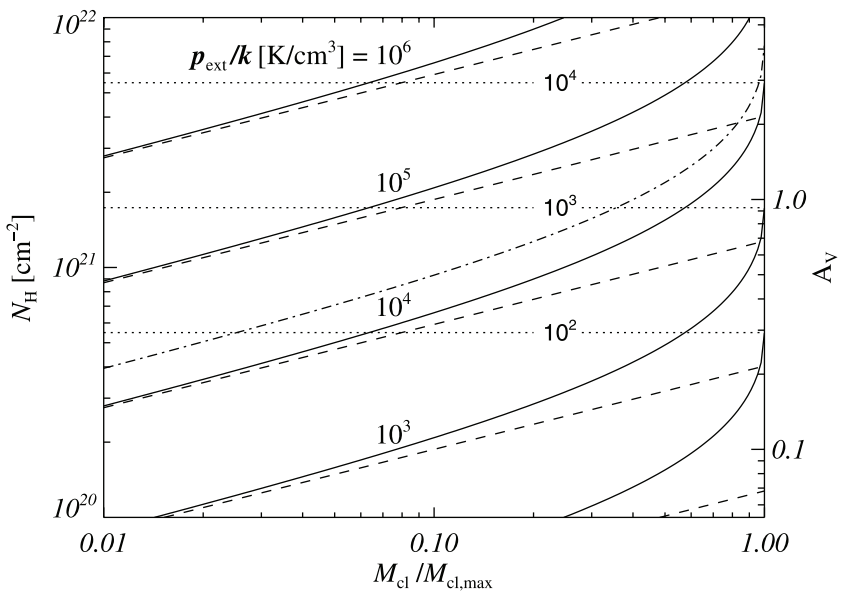

FIG. 4.-Column density and extinction $A_{V}$ toward the center of gravitationally stable isothermal clouds as a function of their critical mass fraction $f$. The extinction in $V$ is obtained by using a gas-to-dust ratio, $N_{\mathrm{H}}=5.8 \times 10^{21} E(B-V)$ (Bohlin et al. 1978), and adopting a value of $R_{V}=3.1$ for the absolute to relative extinction, derived from the observed mean extinction curve of our Galaxy (Fitzpatrick 1999). The column density depends on the gas pressure of the ambient medium, which is varied over several magnitudes up to $p_{\text {ext }} / k=10^{6} \mathrm{~K} \mathrm{~cm}^{-3}$. The dash-dotted line corresponds to a pressure of $p_{\text {ext }} / k=2 \times 10^{4} \mathrm{~K} \mathrm{~cm}^{-3}$, appropriate for the ISM of our Galaxy. For comparison the column densities through homogeneous clouds is also shown (dashed lines). The maximum column density is shown as horizontal dotted lines labeled with the corresponding pressure $p_{\text {ext }} / k$ of the ambient medium.

clouds given by van Dishoeck \& Black (1988). In higher pressure regions such as in starburst galaxies or H II regions these isothermal self-gravitating clouds are expected to be optically thick.

The steeper radial density profile for higher critical mass fractions provides a steeper profile of the column density, as shown in Figure 5. Clouds close to the critical mass against gravitational collapse have column densities through the central region almost 6 times higher than through a homogeneous sphere of the same dimension and outer density.

In the frame of the model we can classify the different cloud types. Translucent clouds characterized by an $A_{V}$ from 1 to $\sim 5$ in the WNM with $p_{\text {ext }} / k=2 \times 10^{4} \mathrm{~K} \mathrm{~cm}^{-3}$ would typically have a mass in the range from 0.1 to 0.9 of the critical mass. Clouds with less than $10 \%$ of the critical mass are diffuse, and clouds with a mass fraction of more than $\approx 90 \%$ are dense clouds or Bok globules. This shows why globules often have a rather steep density profile that is at the outskirts approximately a power law with $n_{\mathrm{H}} \propto$ $r^{-2}$ while translucent clouds typically have a flatter density profile.

\section{THE DUST MODEL}

In this paper we assume that the dust properties inside the isothermal clouds are the same as the mean properties of the diffuse ISM of our Galaxy. For the translucent clouds with $A_{V}<3$ this might be a very good approximation. However, we do not expect this to be the case for more compact clouds. In these, grain growth processes probably play an important role (Whittet et al. 2001), and our assumption about the grain properties in those clouds could well break down.

The dust is assumed to have a certain composition and size distribution. The number of grains of composition $i$ in the radius interval $[a, a+d a]$ is given by $\operatorname{dn}(a)=\operatorname{da} n_{\mathrm{H}} \zeta_{i} f_{i}(a)$, where $n_{\mathrm{H}}$ is the number density of hydrogen atoms and $\zeta_{i}$ is a constant that we have chosen for the abundance of a key element condensed in grain species $i$. We consider a mixture of silicate, graphite, and iron grains and additional PAH molecules. The dust grains composed of silicate, graphite, and iron are assumed to have a

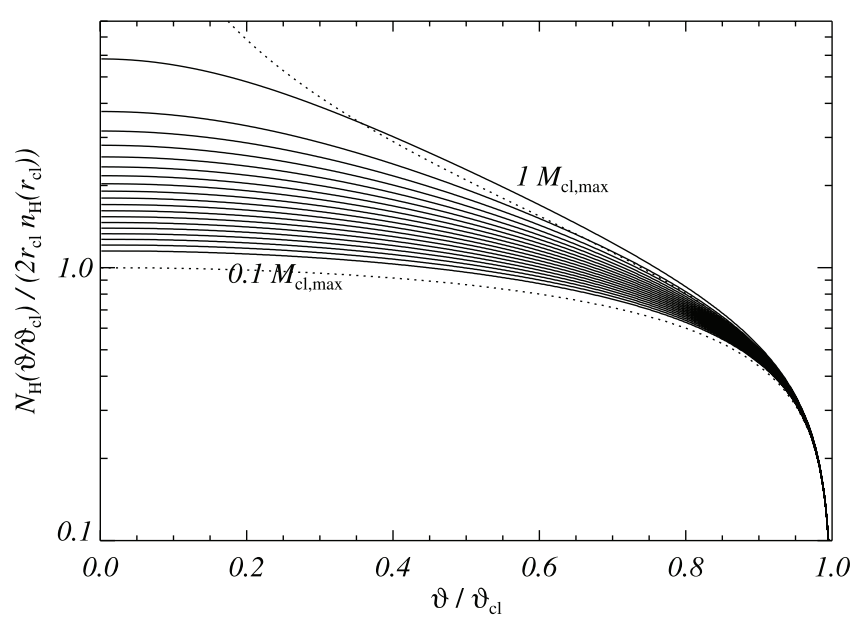

FIG. 5.-Profile of the column densities through distant isothermal clouds $\left(\vartheta_{\mathrm{cl}}=r_{\mathrm{cl}} / D \ll 1\right.$, where $r_{\mathrm{cl}}$ and $D$ are the cloud radius and the distance to the cloud center, respectively). The column density is normalized to $2 r_{\mathrm{cl}} n_{\mathrm{H}}(\mathrm{cl})$. The profile depends on the critical mass fraction $f=M_{\mathrm{cl}} / M_{\mathrm{cl}, \max }$, which is varied from 0.1 to 1.0 in steps of 0.05 . The dotted lines show the profiles for a homogeneous sphere (lowest curve) and a sphere with a steep density profile $\rho \propto r^{-2}$.

power-law distribution with smooth exponential cutoffs at both small and large grain sizes:

$$
\ln \tilde{f}_{i}(a)=-k_{i} \ln a-\left(\frac{a_{i, \min }}{a}\right)^{m_{1}}-\left(\frac{a}{a_{i, \max }}\right)^{m_{2}} .
$$

For PAH molecules we assumed a simple lognormal density distribution:

$$
\ln \tilde{f}_{\mathrm{PAH}}(a)=-\ln a-\frac{1}{2 \sigma_{\mathrm{PAH}}^{2}}\left(\ln a-\ln a_{0}\right)^{2},
$$

with $\ln a_{0}=\ln \left\langle a_{\mathrm{PAH}}\right\rangle-(1 / 2) \sigma_{\mathrm{PAH}}^{2}$, where $\left\langle a_{\mathrm{PAH}}\right\rangle$ and $\sigma_{\mathrm{PAH}}$ are the mean grain size and the standard deviation, respectively.

The distribution functions $\tilde{f}_{i}(a)$ are normalized to the expression

$$
f_{i}(a)=\frac{m_{u}}{(4 / 3) \pi \int \tilde{f}_{i}(a) a^{3} d a}\left\langle\frac{\mu}{\rho}\right\rangle_{i} \tilde{f}_{i}(a)
$$

to obtain the correct units for $\zeta_{i}$. Here $m_{\mathrm{u}}$ is the atomic mass unit, and $\langle\mu / \rho\rangle_{i}$ is the averaged ratio of the mean atomic mass $\mu_{i}$ of the chemical composition and the density $\rho_{i}$ of the grain.

Following Li \& Draine (2001), the emission features of the PAH molecules differ between charged and uncharged states. To determine the SED of isothermal clouds, we assumed the probability of ionized PAH molecules as given for the CNM; see Figure 7 of Li \& Draine (2001).

The parameters for the grain composition and for the size distribution are obtained by fitting simultaneously the mean extinction of our Galaxy (closely consistent with Fitzpatrick 1999 and Martin \& Whittet 1990), the diffuse IR emission measured with the Diffuse Infrared Background Experiment (DIRBE) on board the Cosmic Background Explorer (COBE) satellite (Arendt et al. 1998), and the depletion of key elements (C, O, Si, Mg, and Fe) from the gas phase (Kimura et al. 2003). The depletion is generally obtained by comparing the abundance in the gas phase with the abundances derived for the Sun or other stars' photospheres using radiative transfer codes. The actual abundance of the gas phase depends on the ionization fraction of the gas. Kimura et al. provide two depletion factors for an ionization fraction of 0.25 and 0.45 . We adopted the values for 0.45 . The depletion of the 
TABLE 1

Physical Grain Parameters

\begin{tabular}{|c|c|c|}
\hline Composition & $\begin{array}{l}\text { Density } \rho_{i} \\
\left(\mathrm{~g} \mathrm{~cm}^{-3}\right)\end{array}$ & $\begin{array}{l}\text { Atomic Weight } \mu_{i} \\
(\mathrm{amu})^{\mathrm{a}}\end{array}$ \\
\hline 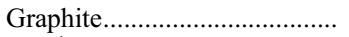 & 2.24 & 12.01 \\
\hline $\mathrm{PAH}^{\mathrm{b}}$ & 2.24 & 12.01 \\
\hline Iron & 7.53 & 55.85 \\
\hline Fosterite $\left(\mathrm{Mg}_{2} \mathrm{SiO}_{4}\right) \ldots \ldots \ldots \ldots \ldots$ & 3.21 & 140.694 \\
\hline Fayalite $\left(\mathrm{Fe}_{2} \mathrm{SiO}_{4}\right) \ldots \ldots \ldots \ldots \ldots$ & 4.30 & 203.776 \\
\hline 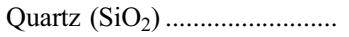 & 2.2 & 60.08 \\
\hline
\end{tabular}

a Atomic mass units. atoms.

elements of interest in this work are based on the solar abundances as given by Holweger (2001). The values are consistent with those given by Zubko et al. (2004).

The actual composition of silicate grains is not certain, but it seems that silicates have generally a low iron content (Gail \& Sedlmayr 1999). In our model we considered silicates to be a mixture of magnesium-rich olivine [itself a mixture between fosterite $\left(\mathrm{Mg}_{2} \mathrm{SiO}_{4}\right)$ and fayalite $\left(\mathrm{Fe}_{2} \mathrm{SiO}_{4}\right)$ summarized as $\left.\mathrm{Mg}_{2 x} \mathrm{Fe}_{2(1-x)} \mathrm{SiO}_{4}\right]$ and quartz $\left(\mathrm{SiO}_{2}\right)$. For olivine we used $x=$ 0.7 , consistent with theoretical calculations about the formation of dust grains in the winds of oxygen-rich stars (Gail \& Sedlmayr 1999). For simplicity we assume the same optical properties for the different silicate compositions.

The assumed physical grain properties are summarized in Table 1. The optical properties for silicate and graphite grains are taken from Draine \& Lee (1984), Laor \& Draine (1993), and Weingartner \& Draine (2001) and those for nonionized and ionized PAH molecules from Draine \& Li (2007). For iron grains we adopted the optical properties given by Fischera (2004).

In the calculation of the thermal reemission by dust grains, we accurately take into account the stochastic heating of the grains by the ambient radiation field. Large grains maintain a certain temperature determined by the equilibrium of heating and cooling rates. However, in the case of small grains where the thermal energy is typically smaller than the energy of the absorbed photon, the stochastic heating can lead to large temperature fluctuations.

The probability distribution for the temperature of small grains is obtained by adopting the method described by Voit (1991), which is a combination of the numerical integration of Guhathakurta \& Draine (1989) and a stepwise analytic approximation. This method avoids inaccuracies of the pure numerical integration in the limit of small temperature fluctuations in the transition from the behavior of small to large grains and allows a relatively small number of integration intervals.

The grain model calculation implies for every grain size and composition equilibrium between absorbed and emitted radiation. The energy is conserved to better than $2 \%$, where the largest dis- crepancy occurs for smallest grain sizes. The individual grain spectra are scaled to have the energy balance fulfilled.

The heat capacities for silicate, graphite, and iron grains needed to derive the temperature probability distributions are summarized in Fischera (2000). For PAH molecules we used the corrected formula for the thermal heat given by Li \& Draine (2001). ${ }^{2}$

The assumed model parameters obtained through a combined fit are summarized in Table 2. The fraction of condensed silicon in olivine is 0.74 . The mean optical properties for our grain composition and size distribution are shown in Figure 6. We note the following principal features:

1. The feature in the extinction curve at $2200 \AA$ is entirely explained by the presence of PAH molecules.

2. The size distribution of carbon grains (PAH molecules and graphite grains) is bimodal. The PAH molecules have a narrow distribution around $6.5 \AA$, while the graphite grains are restricted to the relatively large grains $a>0.01 \mu \mathrm{m}$, which do not show a strong absorption feature at $2200 \AA$. This result is similar to that found by Zubko et al. (2004) for a mixture composed of silicates, graphites, and PAH molecules and strengthens the association of the $2200 \AA$ absorption feature with PAH molecules, rather than with graphite.

To explain the diffuse emission at $60 \mu \mathrm{m}$, we introduced iron grains, which were also proposed to explain the $60 \mu \mathrm{m}$ emission measured with IRAS (Chlewicki \& Laureijs 1988). The size distribution for iron grains is chosen to be the same power-law distribution as for silicon grains but with a slightly smaller maximum grain size. A different assumption for iron grains (allowing smaller iron grains) could well modify the shape of the excess and could even reduce the amount of iron necessary to be condensed in iron grains.

The model parameters are somewhat different to those given by Weingartner \& Draine (2001), which leads to differences in the mean optical properties. Even though the mean extinction curve is similar, our dust model predicts higher absorption relative to the scattering. In addition the scattered light in the UV/ optical/NIR is somewhat less forward scattered.

\section{THE RADIATIVE TRANSFER CODE}

The code used to derive the SED of isothermal clouds will be fully described in a separate paper. Here we summarize its main features.

The code is quite general and can be applied both to clouds that are heated only from outside by an isotropic radiation field or

\footnotetext{
2 There is a mistake in their recommended formula for the thermal heat, a two-dimensional Debye model with slightly different Debye temperatures relative to the original work by Krumhansl \& Brooks (1953). In the formula the dimension needs to be in the numerator but appears in the denominator instead so that the thermal heat is underestimated by a factor of 4 . It appears that the authors were using the correct formula as it would have produced too much emission at short wavelengths.
}

TABLE 2

Grain Model Parameters

\begin{tabular}{|c|c|c|c|c|c|c|c|c|}
\hline Composition & $\begin{array}{c}\zeta_{i} \\
n_{i} / n_{\mathrm{H}} \\
\left(10^{-6}\right)\end{array}$ & $k$ & $\begin{array}{c}a_{\min } \\
(\mu \mathrm{m})\end{array}$ & $\begin{array}{l}a_{\max } \\
(\mu \mathrm{m})\end{array}$ & $m_{1}$ & $m_{2}$ & $\begin{array}{c}\left\langle a_{\mathrm{PAH}}\right\rangle \\
(\mathrm{nm})\end{array}$ & $\sigma_{\ln a_{\mathrm{PAH}}}$ \\
\hline Silicate $\ldots \ldots \ldots \ldots \ldots \ldots \ldots \ldots \ldots$ & 33.22 & 3.5 & 0.001 & 0.257 & 3 & 3 & & \\
\hline Graphite............................ & 199.9 & 5.5 & 0.070 & 1.0 & 3 & 3 & & \\
\hline Iron & 11.14 & 3.5 & 0.001 & 0.1 & 3 & 3 & & \\
\hline PAH & 72.82 & & & & & & 0.650 & 0.119 \\
\hline
\end{tabular}



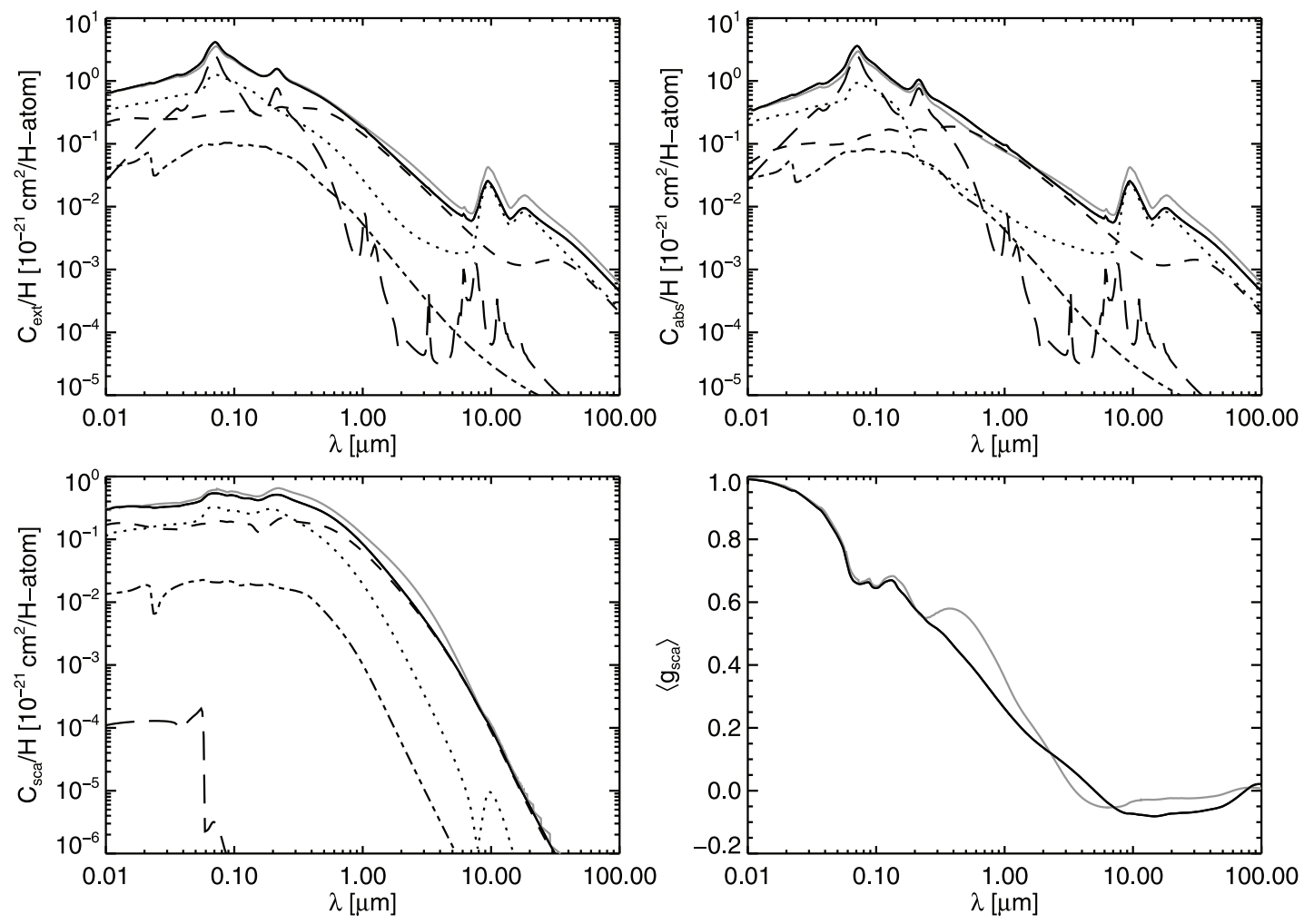

FIG. 6.- Mean optical properties of the dust grains inside the clouds (black solid lines). The data are compared with the mean properties obtained by Weingartner \& Draine (2001) (gray solid lines). Their values for the extinction, absorption, and scattering have been corrected by a factor of 0.93 . The contribution of different dust compositions to the mean values are shown for graphites (dashed lines), silicates (dotted lines), PAH molecules (long-dashed lines), and iron grains (dash-dotted lines).

to shells heated both from a central source and an outer radiation field. In the code the light of the sources and the scattered and emitted light is treated separately to allow a quantitative analysis of the importance of the different components. Here, we follow the nomenclature used in the code. The light of the internal source and the external source are labeled with the index " 1 " and " 4 ," respectively, and the scattered light and the emitted light are labeled with " 2 " and " 3 ," respectively.

To solve the radiative transfer problem including reemission from dust grains, a ray-tracing code is used because it allows a realistic determination of the light distribution of the external light $I_{\nu}^{(4)}(r, \vartheta)$, the scattered light $I_{\nu}^{(2)}(r, \vartheta)$, and the emitted light $I_{\nu}^{(3)}(r, \vartheta)$ inside the cloud. Anisotropic scattering and multiple scattering events are both taken accurately into account. To properly account for reheating by dust grains and scattering of reemitted photons, the problem is solved iteratively. Polarization is considered to be unimportant in determining the form of the SED and is therefore neglected.

The cloud is divided into $N$ shells of constant density that are each optically thin to the attenuated radiation. Therefore, in the case of very compact clouds the column density through the optically thin shells increases inwards. We used a maximum optical thickness relative to the attenuated flux density of $\tau=0.3$.

To probe the angular distribution of the light inside individual shells, we used the mean distances $\left\langle r_{i}\right\rangle$ of each thin shell $i$ to the cloud center as the rays' impact parameters (that is, the closest distance achieved by a given ray from the cloud center). One further ray is added with impact parameter zero. The angular information is obtained for each ray as they cross the thin shells at different angles. The light distribution in shell $i$ is derived by calculating the light along $i+1$ rays. Since rays interior to a shell cross the shell twice, they provide brightness information both for light crossing the shell from outside to the inside and for the light crossing the shell from inside to the outside. One ray provides the brightness of the light that passes the shell tangentially. Using this method, we obtain for shell $i$ the brightness information at $2 i+1$ different angles.

Absorption and scattering are derived using the averaged optical properties in the shells. To calculate anisotropic scattering, we made use of the Henyey-Greenstein scattering function (Henyey $\&$ Greenstein 1941) that is a smooth angular function of the scattering angle and that depends only on the $g$-parameter, the cosineweighted mean of the scattering function of unpolarized light. The scattering problem is solved by deriving successively the brightness distribution $I_{\nu}^{[2(s)]}(r, \vartheta)$ produced by photons scattered 1,2 , $3, \ldots, s$ times.

We consider the flux of scattering events as unimportant if the fractional increase of the total luminosity of scattered photons in all shells is less than $10^{-3}$. In general the maximum number of scattering events taken into account is less than 10 in all calculations. The iterative process terminates if the increase in luminosity per additional scattering is also less than $10^{-3}$.

As a main result the radiative transfer code provides the intensities $I_{\nu}^{(n)}\left(r_{\mathrm{cl}}, \vartheta\right)$ of the attenuated flux $(n=4)$, the scattered flux $(n=2)$, and the flux of the dust emission $(n=3)$ at the cloud radius $r_{\mathrm{cl}}$. In addition it also provides the scattered intensities $[n=2(s)]$ of photons scattered exactly $s$ times before leaving the cloud. The flux density of the different components leaving the cloud is given by

$$
F_{\nu}^{-(n)}=2 \pi \int_{0}^{\pi / 2} d \vartheta \sin \vartheta \cos \vartheta I_{\nu}^{(n)}\left(r_{\mathrm{cl}}, \vartheta\right),
$$


TABLE 3

Cloud Parameters $^{\mathrm{a}}$

\begin{tabular}{|c|c|c|c|c|c|c|c|c|c|}
\hline $\begin{array}{c}M_{\mathrm{cl}} \\
\left(M_{\mathrm{cl}, \text { max }}\right)\end{array}$ & $\begin{array}{c}p_{\text {ext }} / k \\
\left(\mathrm{~K} \mathrm{~cm}^{-3}\right)\end{array}$ & $\begin{array}{c}M_{\mathrm{cl}} \\
\left(M_{\odot}\right)\end{array}$ & $\begin{array}{l}r_{\mathrm{cl}} \\
(\mathrm{pc})\end{array}$ & $\begin{array}{l}n_{\mathrm{H}}(0) \\
\left(\mathrm{cm}^{-3}\right)\end{array}$ & $\begin{array}{c}N_{\mathrm{H}}^{\mathrm{b}} \\
\left(10^{21} \mathrm{~cm}^{-2}\right)\end{array}$ & $\tau_{V}^{\mathrm{b}}$ & $N_{\text {shell }}{ }^{\mathrm{c}}$ & $N_{\text {sca }}{ }^{\mathrm{d}}$ & $\begin{array}{c}\text { Accuracy } \\
(\%)\end{array}$ \\
\hline $0.500 \ldots \ldots \ldots \ldots \ldots \ldots$ & $1 \times 10^{3}$ & 574.2 & 5.582 & 36.99 & 0.489 & 0.225 & 5 & 4 & 0.01 \\
\hline $0.001 \ldots \ldots \ldots \ldots \ldots$ & $2 \times 10^{4}$ & 0.257 & 0.174 & 336.4 & 0.180 & 0.083 & 2 & 4 & -0.17 \\
\hline $0.010 \ldots \ldots \ldots \ldots \ldots \ldots$ & $2 \times 10^{4}$ & 2.568 & 0.373 & 347.8 & 0.395 & 0.182 & 4 & 4 & 0.02 \\
\hline $0.100 \ldots \ldots$ & $2 \times 10^{4}$ & 25.68 & 0.786 & 412.4 & 0.932 & 0.429 & 9 & 5 & 0.09 \\
\hline $0.500 \ldots \ldots \ldots \ldots \ldots \ldots \ldots$ & $1 \times 10^{5}$ & 57.42 & 0.558 & 3699 & 4.895 & 2.251 & 44 & 8 & 0.09 \\
\hline $0.500 \ldots \ldots$ & $1 \times 10^{6}$ & 18.16 & 0.177 & $3.70 \times 10^{4}$ & 15.48 & 7.117 & 139 & 9 & 0.11 \\
\hline 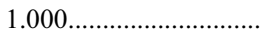 & $1 \times 10^{6}$ & 36.32 & 0.182 & $2.34 \times 10^{5}$ & 54.88 & 25.23 & 353 & 9 & 0.10 \\
\hline
\end{tabular}

a The cloud temperature is chosen be $100 \mathrm{~K}$ and the mean molecular weight 2.36 .

${ }^{\mathrm{b}}$ Column density and optical depth measured to the cloud center.

${ }^{c}$ Number of shells used to solve the radiative transfer problem.

${ }^{d}$ Number of scattering events taken into account.

e Determined as the total flux (attenuated flux, scattered flux, and reemitted flux) leaving the cloud relative to the flux entering the cloud.

where $n=4,3,2$, or $2(s)$. The flux density entering the cloud is given by

$$
F_{\nu}^{+(4)}=\pi J_{\nu}^{\mathrm{ISRF}},
$$

where $J_{\nu}^{\mathrm{ISRF}}$ is given by equation (9). The corresponding integrated flux has to be equal to the scattered, the reemitted, and the attenuated light leaving the cloud:

$$
F^{+(4)}=F^{-(2)}+F^{-(3)}+F^{-(4)} .
$$

We have used this relation to verify our calculations.

\section{MODEL CALCULATIONS AND RESULTS}

In the following we show the effects of the critical mass fraction $f$ and the pressure $p_{\text {ext }} / k$ of the ambient medium of the clouds on the radiative transfer through isothermal clouds. The pressure $p_{\text {ext }} / k$ is varied from $10^{3}$ up to $10^{6} \mathrm{~K} \mathrm{~cm}^{-3}$ and cloud masses from $0.1 \%$ up to $100 \%$ of the critical mass. The clouds are assumed to be heated by the ISRF, which is adopted from Mathis et al. (1982). It is given by

$$
J_{\nu}^{\mathrm{ISRF}}=\chi\left[J_{\nu}^{\mathrm{UV}}+\sum_{i=2}^{4} W_{i} B_{\nu}\left(T_{i}\right)\right]+B_{\nu}(2.7 \mathrm{~K}),
$$

with the dilution factors $W_{2}=10^{-14}, W_{3}=10^{-13}$, and $W_{4}=4 \times$ $10^{-13}$ and temperatures $T_{2}=7500, T_{3}=4000$, and $T_{4}=3000 \mathrm{~K}$. The quantity $J_{\nu}^{\mathrm{UV}}$ is the mean intensity of the UV radiation in the solar neighborhood (Table $\mathrm{C} 1$ of Mathis et al. 1982). This radiation field has a maximum photon energy of $13.6 \mathrm{eV}$. Apart from the ISRF of the local neighborhood $(\chi=1)$, we also assumed a 100 times stronger radiation field $(\chi=100)$.

We assumed a cloud temperature of $100 \mathrm{~K}$. For the gas we adopted the solar abundance of both helium $\left(n_{\mathrm{He}} / n_{\mathrm{H}} \approx 0.1\right)$ and heavier elements and assumed that hydrogen is completely molecular, giving a mean molecular weight of $\mu=2.36$. The mass and the size can be transformed for other conditions using the relations given in $\S 2$. The parameters of the clouds considered in this paper are listed in Table 3 . In addition we give the accuracy of the SED obtained, the number of shells used to solve the ra- diative transfer problem, and the number of scattering events taken into account. The energy conservation (for details see $\S 5.3$ ) in all calculations is better than $0.2 \%$.

First we examine in some detail how the grain heating and grain temperatures vary radially inside the cloud. Then we show how the different parameters affect the mean SED and the brightness profiles at different wavelengths. Most attention is paid to the situation where clouds are embedded in the $\mathrm{WNM}\left(p_{\text {ext }} / k=\right.$ $2 \times 10^{4} \mathrm{~K} \mathrm{~cm}^{-3}$ ) and the mass is chosen to be 0.5 and 1.0 of the critical mass. The results are compared with corresponding calculations for clouds in a much higher pressure region where we have chosen $p_{\text {ext }} / k=10^{6} \mathrm{~K} \mathrm{~cm}^{-3}$.

\subsection{Grain Heating}

To analyze the grain heating in more detail, we have separated the heating rate into the three components of the radiation field inside the cloud: heating by the attenuated external flux, heating by the scattered flux, and finally, heating by the flux of the thermal reemission. The relative contribution to the total heating rate varies as a function of the distance toward the cloud center and depends on the compactness of the clouds and the radial density profile. In Figure 7 the heating rate is shown with distance from the cloud center for different assumptions about the outer pressure and the critical mass fraction. For those calculations we assumed the local ISRF with $\chi=1$.

The variation of the heating as function of radius reflects the different density profiles. For example, in the case of clouds with the critical mass we see a strong decrease close to the cloud center resulting from the steep density profile.

As remarked above, stable clouds embedded in an ISM with $p_{\text {ext }} / k=2 \times 10^{4} \mathrm{~K} \mathrm{~cm}^{-3}$ are not very optically thick. Clouds with a high critical mass fraction of 0.5 have an optical thickness toward the cloud center of only $\sim 0.7$ in $V$. Therefore, the total heating rate in the center of those clouds is less than a factor of 2 lower than in the outskirts.

The heating is predominantly caused by the attenuated external light. For clouds with a small critical mass fraction the scattered light does not make a strong contribution, and the heating by thermal emission from grains is negligible. The contribution of the scattered light to the total heating increases with critical mass fraction. In the central regions of clouds close to the gravitational 

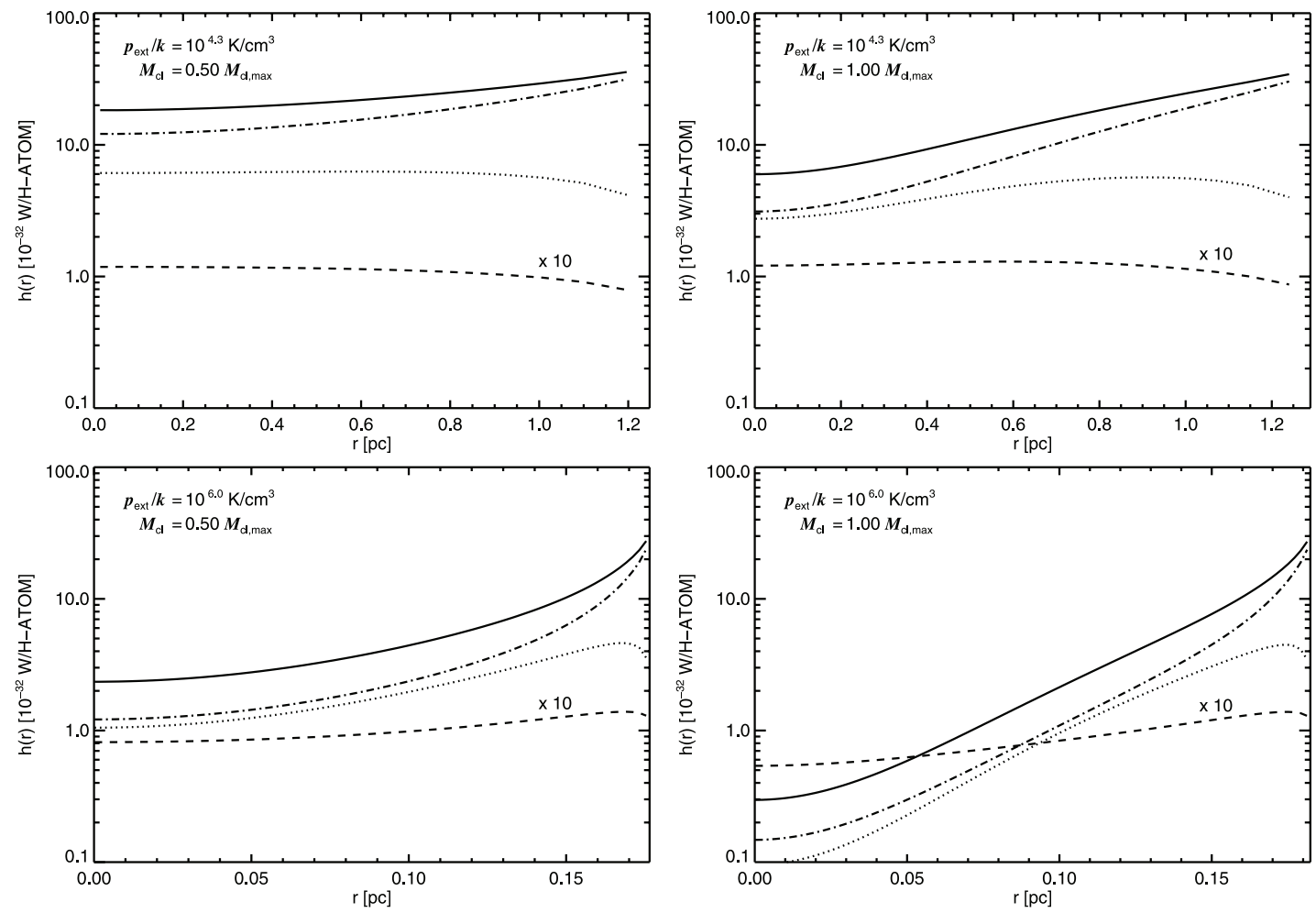

FIG. 7.- Heating rate of the grains as a function of distance from the cloud center for different assumptions of the critical mass fraction and the outer pressure. The clouds are heated by the local ISRF $(\chi=1)$. The cloud temperature is assumed to be $100 \mathrm{~K}$, and the mean molecular weight is taken to be $\mu=2.36$. The total heating rate is shown as a solid line. The heating rates caused by the different components of the radiation, the attenuated external flux, the scattered flux, and the flux of the thermal reemission, are shown as dash-dotted, dotted, and dashed lines, respectively. The heating by the thermal reemission is scaled by a factor of 10 to allow it to be plotted along with the other heating rates.

collapse, the scattered light is almost as important as the attenuated flux.

Clouds in the high-pressure region are 7 times optically thicker. As a result, the total heating decreases by more than a factor of 10 toward the cloud center. Interestingly we found that the scattered light never dominates the heating of the attenuated flux. The heating by the thermal dust emission is significantly higher but still below the other contributions. While the heating by thermal reemission is still unimportant in the center of clouds with $f=$ 0.5 , the contribution increases up to $50 \%$ in the case of critical stable clouds.

The contribution of the thermal emission depends somewhat on the strength of the external radiation field. If the radiation field is more intense, the grains will be warmer, which shifts the radiation of the dust reemission to shorter wavelengths. Because the absorption efficiency of most grains increases toward shorter wavelengths, it therefore becomes more likely that emitted photons are once again absorbed by other dust grains. However, in our models this effect is not very strong. For example, if the radiation field around clouds with $f=0.5$ and $p_{\text {ext }} / k=10^{6} \mathrm{~K} \mathrm{~cm}^{-3}$ is increased by a factor of 100 , the contribution of reemitted photons is increased by less than a factor of 2 .

\subsection{The Grain Temperatures}

The grain temperature distribution inside the clouds determines the spectral shape of the thermal reemission spectrum. A first indication of likely grain temperatures is given by consideration of the radial variation of the heating rate. From the results presented above we would expect that the grain temperature in isothermal clouds that are embedded in a gas with $p_{\text {ext }} / k=2 \times$ $10^{4} \mathrm{~K} \mathrm{~cm}^{-3}$ is not very different from the temperature of grains that are heated directly by the ISRF, unless the clouds are close to collapse.

In detail, the grain temperature is a function of the grain size and composition, as seen in Figure 8. Here we have chosen grains of sufficient size so that they do not show a strong temperature fluctuation and can well be described by a single temperature. For iron we have chosen a size of $0.01 \mu \mathrm{m}$. Because small iron grains do not cool efficiently, their temperatures are generally much larger than for other grain types. We have divided their temperature by a factor of 2 to allow a direct comparison on the plot. One should also be aware that the temperature of iron grains is a strong function of grain size. If the grains are heated by the ISRF, the temperature decreases rapidly toward larger sizes, shows a minimum at $0.25 \mu \mathrm{m}$, and increases slightly to even larger sizes (Fischera 2004). Therefore, the temperature of large iron grains is not necessarily higher than grains of other materials but similar sizes.

The decrease in grain temperature toward the cloud center mainly reflects the decrease of grain heating. But we also see in Figure 8 that the temperatures of small grains decrease more rapidly than the larger grains. The strongest radial variation of temperature is seen for the small iron grains. This effect is caused by the fact that the absorption behavior decreases for wavelengths typically larger than the grain size (or for wavelengths $\lambda>a / 2 \pi$ ). Small grains are efficiently heated by UV radiation. Due to the high extinction at those wavelengths, the UV radiation is strongly reduced, which leads to a strong decrease of the heating of small grains. Large grains are less affected as they are also strongly heated by the optical light.

In case of high optical depths both the UV radiation and the optical radiation are strongly absorbed at the outskirts of the clouds, 

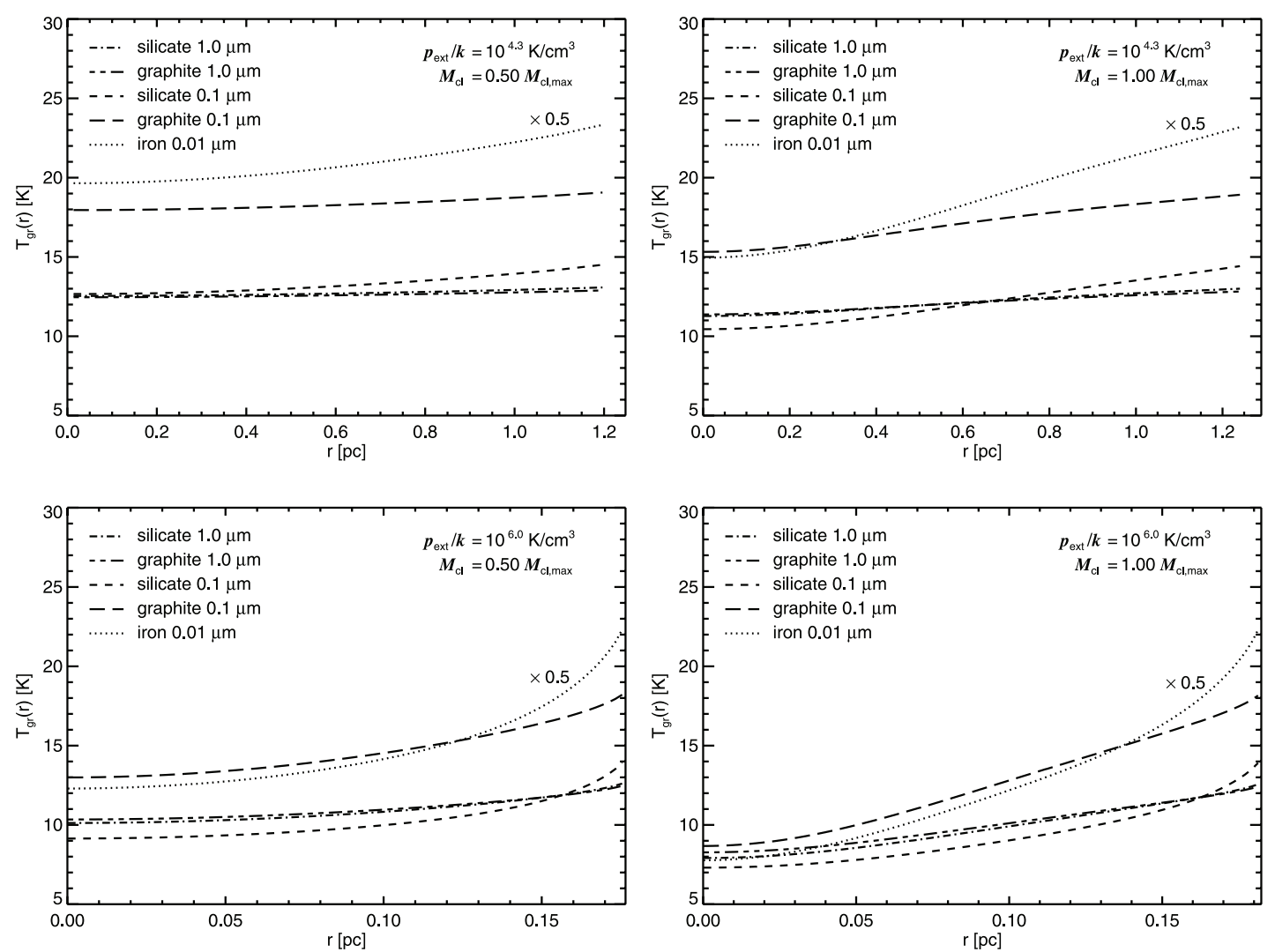

FIG. 8. - Grain temperatures of certain grain sizes and compositions inside isothermal clouds as a function of distance to the cloud center. The temperatures of iron grains have been divided by a factor of 2 .

so the grains in the central region become predominantly heated by IR photons. As the wavelength dependence of the absorption coefficients at those wavelengths is similar for all grains (the absorption coefficient decreases for wavelengths larger than the grain size proportional to $1 / \lambda^{2}$ ), the grain temperatures become equal. However, in all cases the small iron grains show significantly higher temperatures.

We note that for all stable clouds embedded in a medium with $p_{\text {ext }} / k=2 \times 10^{4} \mathrm{~K} \mathrm{~cm}^{-3}$ the grain temperatures lie above $10 \mathrm{~K}$. Colder temperatures can only be produced in collapsing clouds if the assumed grain properties are valid for all critical mass fractions.

\subsection{The SEDs of Isothermal Clouds}

The SED from isothermal clouds heated by the ISRF appropriate to the solar neighborhood is shown in Figure 9. In the near infrared (NIR) the thermal reemission spectrum is dominated by the PAH emission, at wavelengths longer than $\approx 30 \mu \mathrm{m}$ by emission from dust grains. Because the clouds are optically thicker in higher pressure regions, more optical and UV light is transformed to thermal emission in such regions.

The scattered light makes an important contribution to the SED in the optical and UV. If we consider clouds with a high critical mass fraction embedded in a pressure region $p_{\text {ext }} / k=2 \times$ $10^{4} \mathrm{~K} \mathrm{~cm}^{-3}$, roughly half of the flux at wavelengths shorter than $0.5 \mu \mathrm{m}$ is caused by scattered photons. As is shown in $\S 5.4$.1, the contribution of the scattered light to the total flux varies strongly with the line of sight. In higher pressure regions scattered light makes the dominant contribution at wavelengths shorter than $\approx 1 \mu \mathrm{m}$. In addition multiple scattering events become increasingly important.

\subsubsection{The Effect of the Outer Pressure}

As shown above, the clouds are more compact and optically thick in high-pressure regions. One therefore expects more cold dust emission in high-pressure regions if the clouds are heated by the same radiation field. To show the effect of the pressure on the SED, we held the critical mass fraction fixed and only modified the outer pressure. The external radiation was chosen to be the ISRF with $\chi=1$ and $\chi=100$. The result is shown in Figure 10.

The shape of the PAH emission spectrum is mostly determined by the molecular heating associated with the absorption of single high-energy photons and is therefore rather insensitive to the emission spectrum. However, as can be seen, the spectra become slightly less intense at short wavelengths in higher pressure regions because the energetic photons are strongly absorbed in the interior, resulting in less excitation of energetic molecular modes.

As the PAH molecules are predominantly heated by UV and optical light, which is strongly absorbed inside the cloud, their emission relative to the dust emission decreases toward optically thicker clouds. It also shows a shift toward longer wavelengths at higher pressures, as is expected given the lower grain temperatures in the central cloud regions. Such dark clouds may therefore be important contributors to the submillimeter emission of starburst galaxies, which are characterized by higher pressures in the ISM and in which the radiation of compact $\mathrm{H}_{\text {II }}$ regions would otherwise tend to dominate the warmer dust emission (Dopita et al. 2005).

As seen in the figure, for both assumptions of the radiation field we obtain, with the exception of higher grain temperatures and proportional higher emission, quantitatively very similar results 

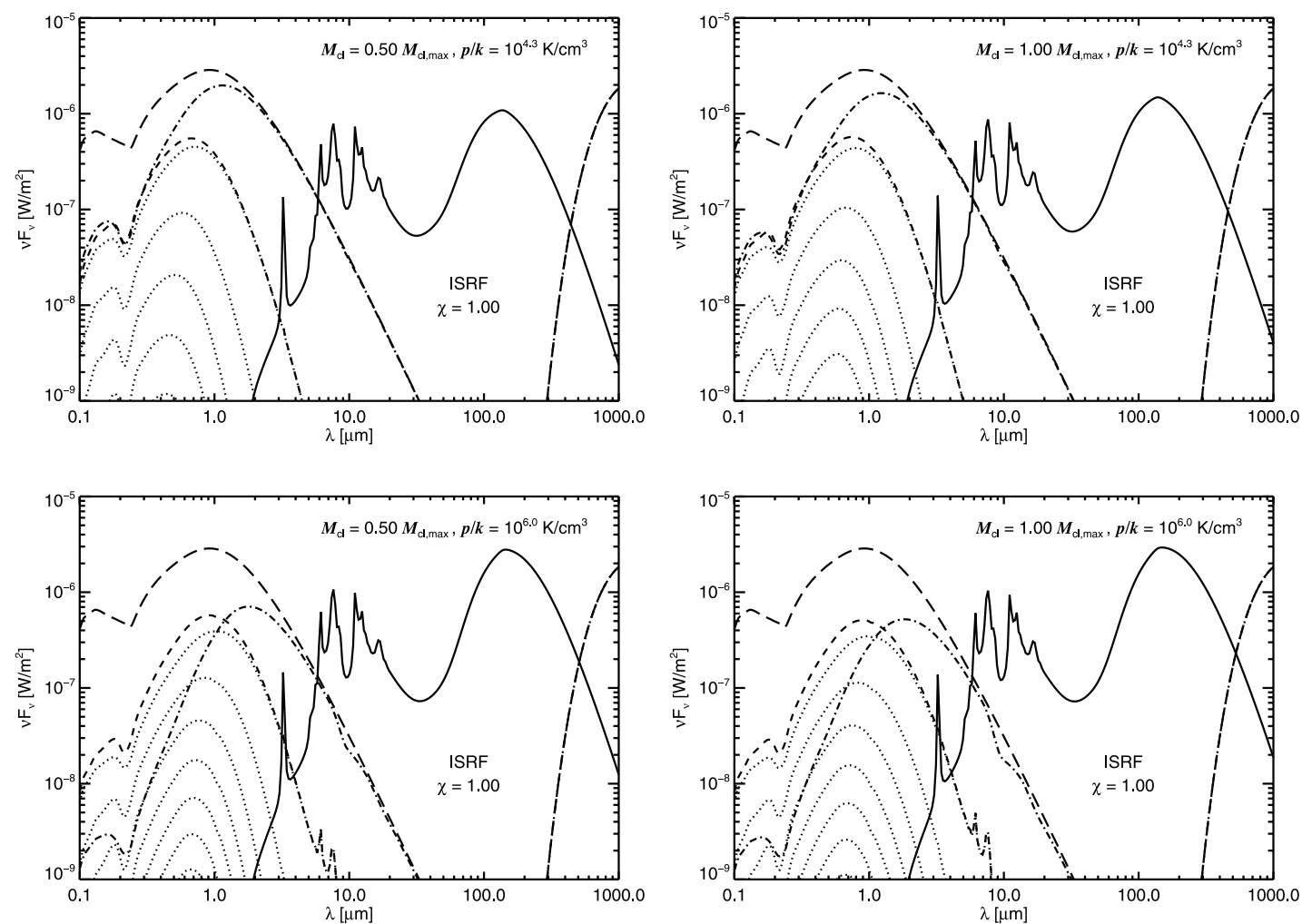

FIG. 9.-SEDs of isothermal clouds with $f=0.5$ and $f=1.0$ heated by the ISRF appropriate to the local neighborhood $(\chi=1)$. The outer pressure $p_{\text {ext }} / k$ is taken to be $2 \times 10^{4}$ and $10^{6} \mathrm{~K} \mathrm{~cm}^{-3}$, respectively. The ISRF entering and heating the cloud is shown as a long-dashed curve. The emission at long wavelengths is the cosmic background radiation. The attenuated external emission leaving the cloud is shown as a dash-dotted line. The scattered radiation is shown as a short-dashed line. This is the sum of all the radiation caused by photons scattered $s$ times (dotted lines). The upper dotted curve is the radiation of photons scattered only once; the dotted curve below that is the radiation of all photons scattered twice, and so on. The thermal reemission spectrum is the solid line.

in the temperature shift and in the ratio between dust and PAH emission.

\subsubsection{The Effect of the Critical Mass Fraction}

A very similar effect in the dust temperature distribution is caused by increasing the critical mass fraction, as one also would also expect for simple homogeneous clouds. Here, the effect is complicated by the change of the radial density profile. We varied the critical mass fraction from 0.1 to 1.0 assuming an outer pressure $p_{\text {ext }} / k=2 \times 10^{4} \mathrm{~K} / \mathrm{cm}^{3}$. The spectra derived for $\chi=1$ and $\chi=100$ are shown in Figure 11 .

\subsection{The Brightness Profiles}

Here we analyze different aspects on the brightness profiles of isothermal clouds. First we present profiles in the optical/NIR regime where the scattered light has a strong contribution to the SED. Then we present brightness profiles of the thermal emission from dust grains and PAH molecules. To provide an angular scale, the clouds have been placed at a distance $D=1 \mathrm{kpc}$ from the observer.

\subsubsection{The Scattered Emission}

For comparison we have chosen wavelengths at 440 and $900 \mathrm{~nm}$, typical for the $B$ and the $I$ filter, respectively. For highly opaque clouds the scattered emission seen by the observer arises predominantly from the outskirts and is therefore not very sensitive to the actual density structure. We see this in Figure 12 for the considered clouds embedded in a pressure medium of $p_{\text {ext }} / k=$ $10^{6} \mathrm{~K} \mathrm{~cm}^{-3}$ where the profiles at $440 \mathrm{~nm}$ are almost identical. At longer wavelengths the same clouds appear optically thinner so
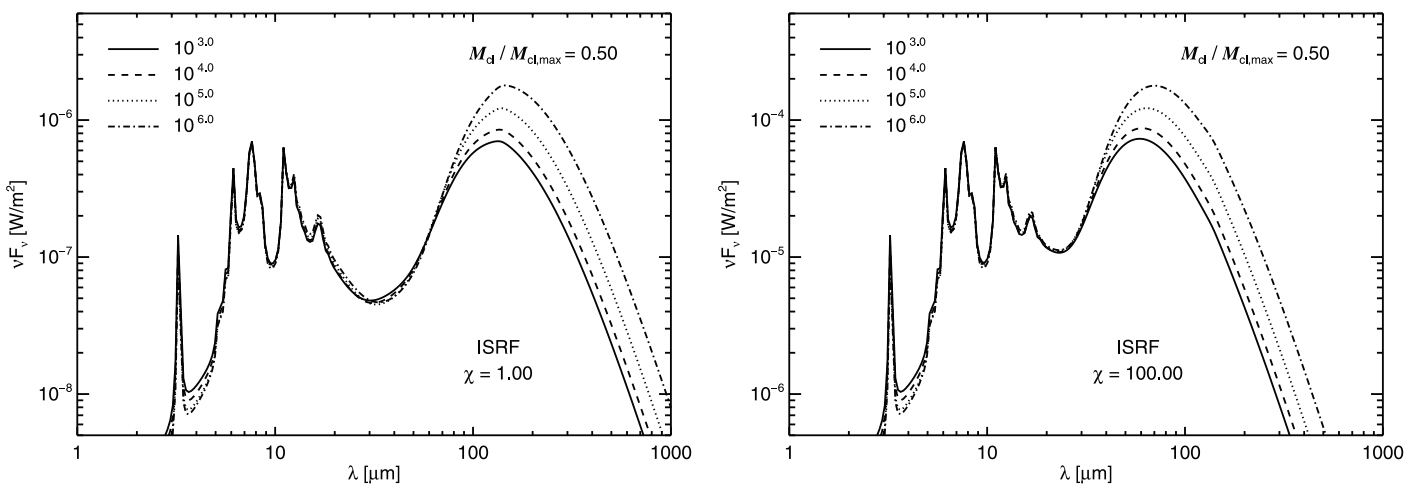

FIG. 10. - Left: Effect of the outer pressure on the SEDs of isothermal clouds heated by the solar vicinity ISRF $(\chi=1.0)$. The critical mass fraction is fixed to $M_{\mathrm{cl}}=$ $0.5 M_{\mathrm{cl} \text { max }}$. The pressure is taken to be $p_{\text {ext }} / k=10^{3}, 10^{4}, 10^{5}$ and $10^{6} \mathrm{~K} \mathrm{~cm}^{-3}$. The spectra are normalized to the emission at $11.3 \mu \mathrm{m}$ in the spectrum for $p_{\text {ext }} / k=10^{4} \mathrm{~K} \mathrm{~cm}{ }^{-3}$. Right: Same clouds as heated by a local radiation field, which is 100 times higher than the local radiation field $(\chi=100)$. 

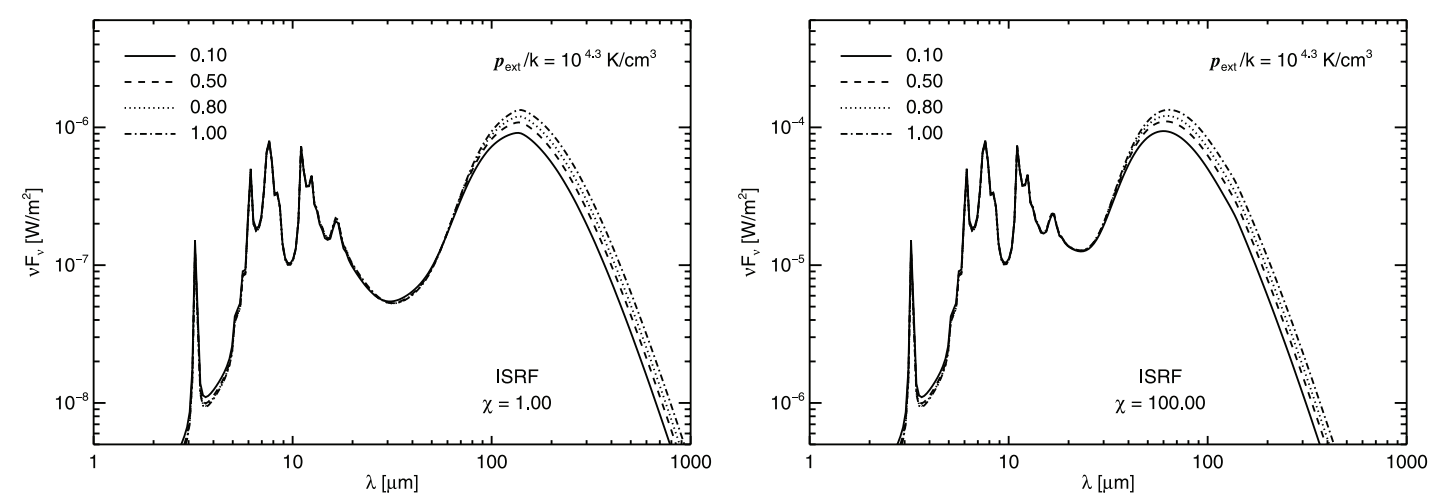

FIG. 11. - Effect of the critical mass fraction on the SED of isothermal clouds. The outer pressure is taken to be $p_{\text {ext }} / k=2 \times 10^{4} \mathrm{~K} \mathrm{~cm}^{-3}$. The critical mass fraction is varied from 0.1 to 1.0 of the maximum stable cloud mass. The spectra are normalized to the emission at $11.3 \mu \mathrm{m}$ relative to the spectrum for $f=0.5$.

that the profiles show some dependence on the mass fraction $f$. We see that the corresponding brightness profiles at $900 \mathrm{~nm}$ show qualitatively a similar shape as at $440 \mathrm{~nm}$, but the emission is less concentrated toward the cloud edges. While the maximum flux is similarly strong at $f=0.5$ and $f=1$, the brightness toward the cloud center decreases with mass fraction $f$ because of the higher probability that the scattered emission is also absorbed in the cloud center.

In the lower pressure region the actual brightness profile depends strongly on the actual density structure of the clouds. While the brightness profile at $440 \mathrm{~nm}$ of low-mass clouds follows qualitatively the column density profile, it develops a clear wing structure toward higher mass fractions, which can be considered as a limb-brightening effect. At longer wavelengths the emission arises more closely from the cloud center, reducing this effect, which at $900 \mathrm{~nm}$ has almost disappeared.

Figure 12 also shows the brightness profiles of photons scattered several times in the cloud before those photons escape. We see that the radial profiles become more centrally concentrated the higher the number of scattering events. The strong concentration in flux toward the outer part of the cloud in the singlescattering case is therefore reduced when we consider the integrated multiple-scattering profile.

\subsubsection{The Thermal Emission}

The brightness profiles at IR and FIR wavelengths provide some insights about the radial variation of the radiation field and of the grain temperatures inside the clouds.

For the purposes of displaying brightness profiles we have chosen six wavelengths covering the range from 8 to $850 \mu \mathrm{m}$. At shorter wavelengths we give the theoretical flux profiles for three different broadband filters of the Spitzer satellite, namely, Infrared Array Camera (IRAC) $4(8 \mu \mathrm{m})$, and Multiband Imaging Photometer for Spitzer (MIPS) 2 and 3 (70 and $160 \mu \mathrm{m}$ ). In the diffuse ISM, they characterize the PAH emission, the warm dust emission, and the cold dust emission, respectively. The three profiles at longer wavelengths, namely, at 250,350, and $850 \mu \mathrm{m}$, are given for narrowband filters. The profiles are normalized to the maximum brightness and compared to the profile of the column density. The results for the two assumed strengths of the radiation fields are shown in Figures 13 and 14.

The brightness $\nu I_{\nu}^{(3)}(0)$ toward the cloud center and the flux density $\nu F_{\nu}^{(3)}$ at the cloud radius are summarized in Table 4 . For completeness we also list the values for the MIPS 1 filter at $24 \mu \mathrm{m}$. Note that in our model the diffuse dust emission at $24 \mu \mathrm{m}$ is dominated by the PAH emission. The flux can therefore not be used to identify warm dust emission.
As mentioned, the PAH molecules in our model are predominantly heated by the UV/optical radiation, which is strongly absorbed inside the cloud. In the case of optically thick clouds $\left(p / k=10^{6} \mathrm{~K} \mathrm{~cm}^{-3}\right)$ the PAH emission is strongest at the outskirts, which causes a limb-brightening effect, as is seen for clouds in the high-pressure medium. The effect is less prominent for clouds embedded in the lower pressure region. A slight minimum in the brightness profile toward the cloud center appears only for clouds close to the critical mass. The maximum of the brightness profile is furthermore not strongly related to the limb as it is located at a position roughly half of the projected radius. As seen in the figure, the brightness profiles of the PAH emission are - as a result of its stochastic nature - almost insensitive to the radiation strength.

The brightness profiles of the thermal emission from dust grains is a result of the decreasing radiation field inside the cloud and the temperature shift of the grains to colder grain temperatures. Naturally they depend on the strength of the external radiation field, as a stronger radiation field produces warmer dust emission.

In the case that the clouds are illuminated by the mean ISRF, the brightness profiles at $70 \mu \mathrm{m}$ show qualitatively the same shape as the PAH emission, although the limb brightening is smaller and the emission peak is closer to the cloud center.

In the low-pressure region the brightness profiles at $170 \mathrm{~nm}$ follow more closely the qualitative profile of the column density with a prominent maximum toward the cloud center for critically stable clouds. In the high-pressure environment the profile produces a winglike shape because of the cold dust emission from the cloud center.

In the case of a 100 times stronger radiation field the diffuse dust emission has a peak around $70 \mu \mathrm{m}$. For the corresponding brightness profiles at $70 \mu \mathrm{m}$ we obtain qualitatively the same shapes as for the lower radiation field at $170 \mu \mathrm{m}$.

The brightness profiles at long wavelengths appear always broader in comparison with the profile of the column density. The difference is larger for shorter wavelengths and more prominent for optically thicker clouds. In general the effect decreases for higher radiation fields.

\section{DISCUSSION}

The model we have presented here relates the SED from selfgravitating clouds to the physical properties of the diffuse inhomogeneous ISM. Even though it is based on a simplified structure of the compact clouds, it might find application in helping us to understand the SED of whole galaxies.

We have seen that clouds in the ISM of our Galaxy are essentially optically thin unless they are close to the critical mass or 

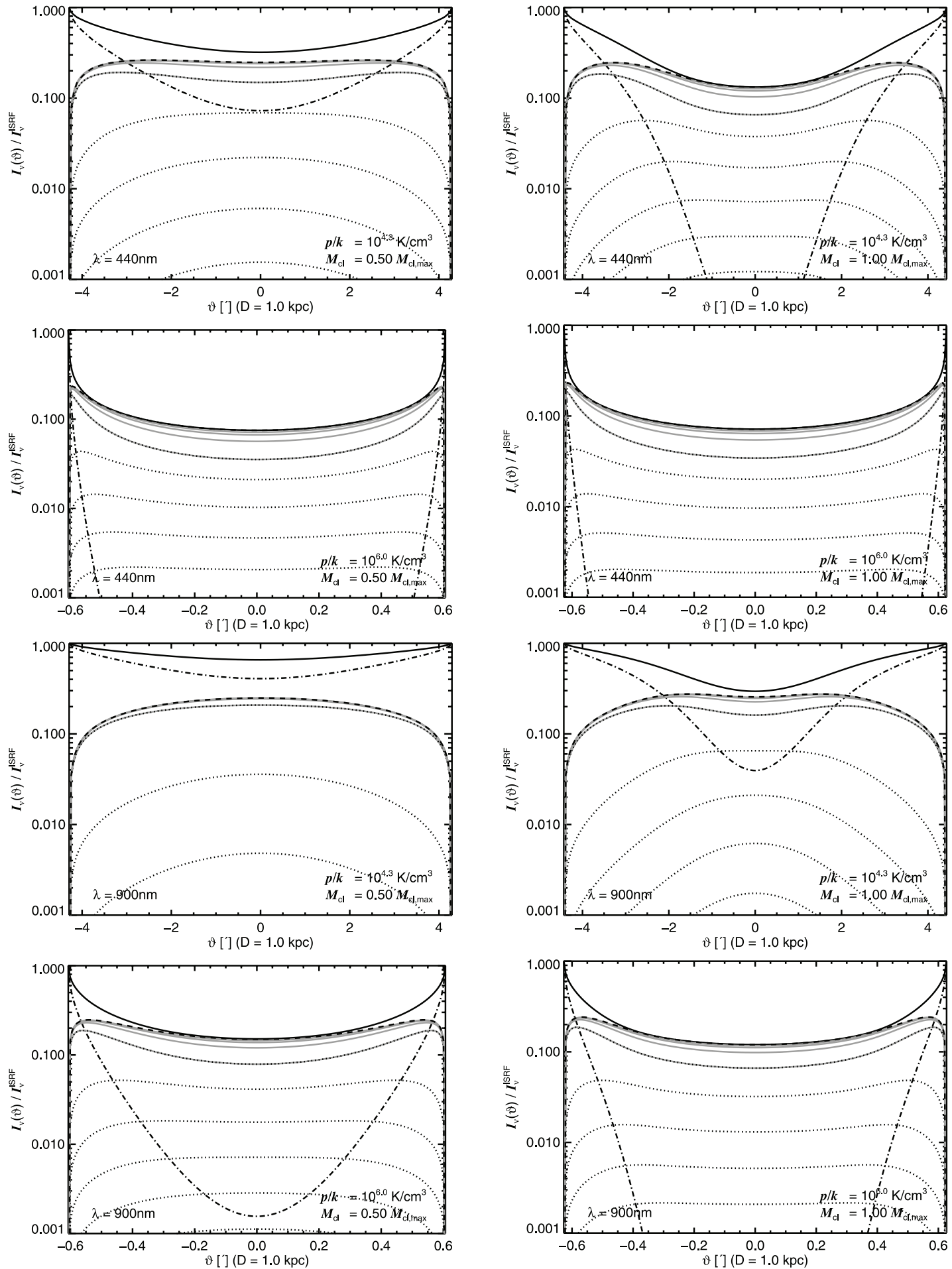

FIG. 12.-Brightness profiles at $440 \mathrm{~nm}$ (top four panels) and $900 \mathrm{~nm}$ (bottom four panels) for isothermal clouds located at a distance of $1 \mathrm{kpc}$ heated by the ISRF. The pressure $p_{\text {ext }} / k$ is chosen to be $2 \times 10^{4}$ and $10^{6} \mathrm{~K} \mathrm{~cm}^{-3}$, while the mass fraction $f$ is assumed to be either 0.5 or 1.0 . Shown are the brightness profiles of the total flux (solid line), the scattered flux (dashed line), and the attenuated external flux (dash-dotted line). The dotted lines are brightness profiles of photons scattered $s$ times before they escape from the dust cloud. The brightness profiles corresponding to the photons scattered at maximum $s$ times are shown for comparison as gray lines.

are in a state of collapse. The effect will depend on how much dust mass is actually distributed in those clouds. In our galaxy gravitational bound clouds should be closely related to the molecular gas as they are sufficiently opaque against UV-radiation. According to Ferrière (2001, Table 1) the molecular mass is probably for our galaxy not larger than $20 \%$ of the ISM. The effect of the inhomogeneous density structure on the global SED of our or any normal galaxy should therefore be quite limited. A simplified model is presented in $\S 6.1$ to quantify the effect.

The situation might be very different in star-forming regions or starburst galaxies, where the pressure of the diffuse ISM might be significantly higher (Dopita et al. 2005) so that the stable clouds will be more compact. In those cases the inhomogeneous structure of the ISM should have a bigger effect on the SED as also nongravitational bound clouds can be optically thick. 

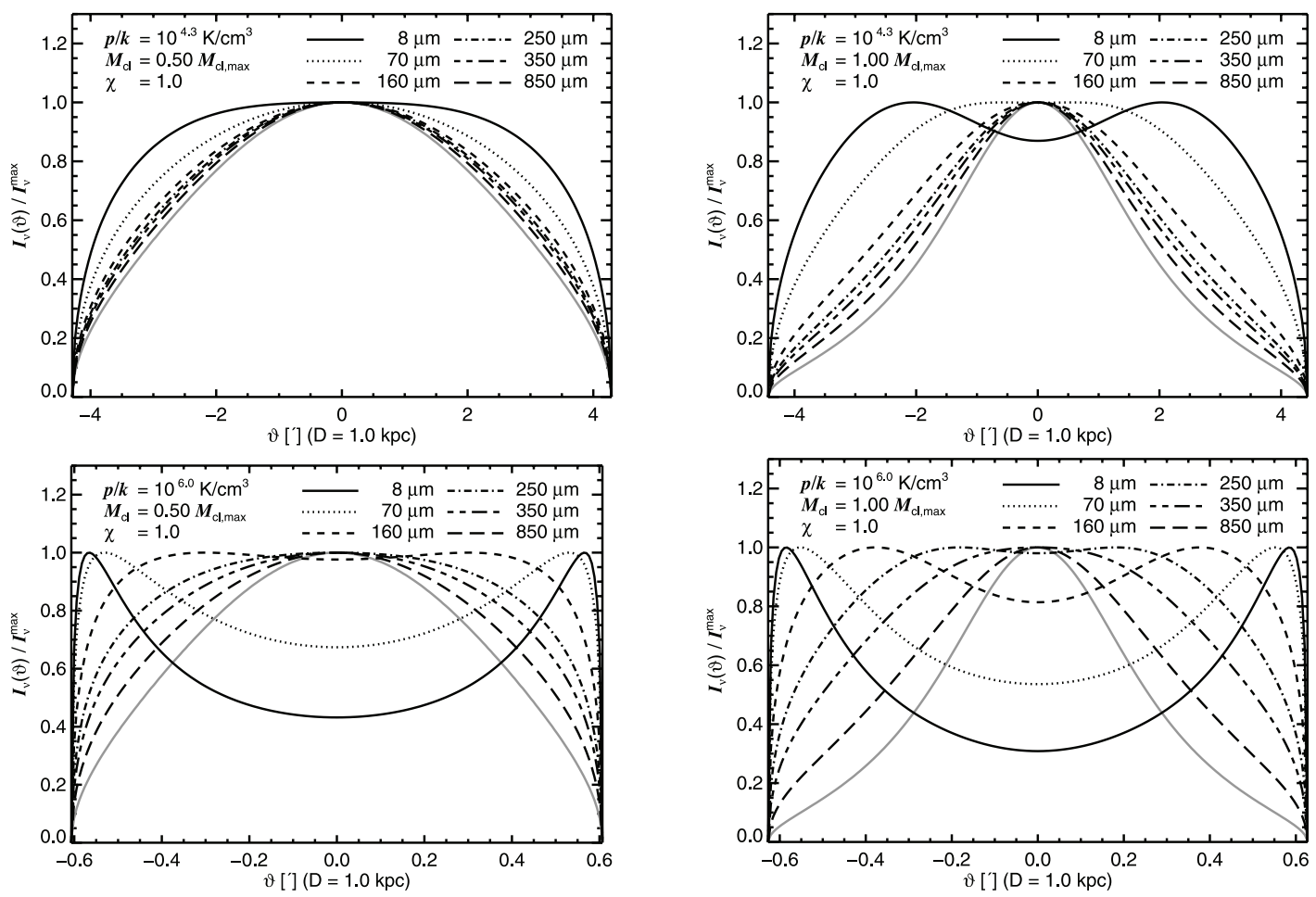

FIG. 13. - Normalized brightness profiles at several IR wavelengths of isothermal clouds, which are located at a distance of $1 \mathrm{kpc}$ and heated by the local ISRF $(\chi=1)$. The profiles are compared with profiles of the column density (gray lines). The outer pressure is assumed to be $p_{\text {ext }} / k=2 \times 10^{4} \mathrm{~K} \mathrm{~cm}^{-3}$ (top) and $p_{\text {ext }} / k=10^{6} \mathrm{~K} \mathrm{~cm}{ }^{-3}$ (bottom). The mass fraction is taken to be 0.5 and 1.0 (left and right, respectively).
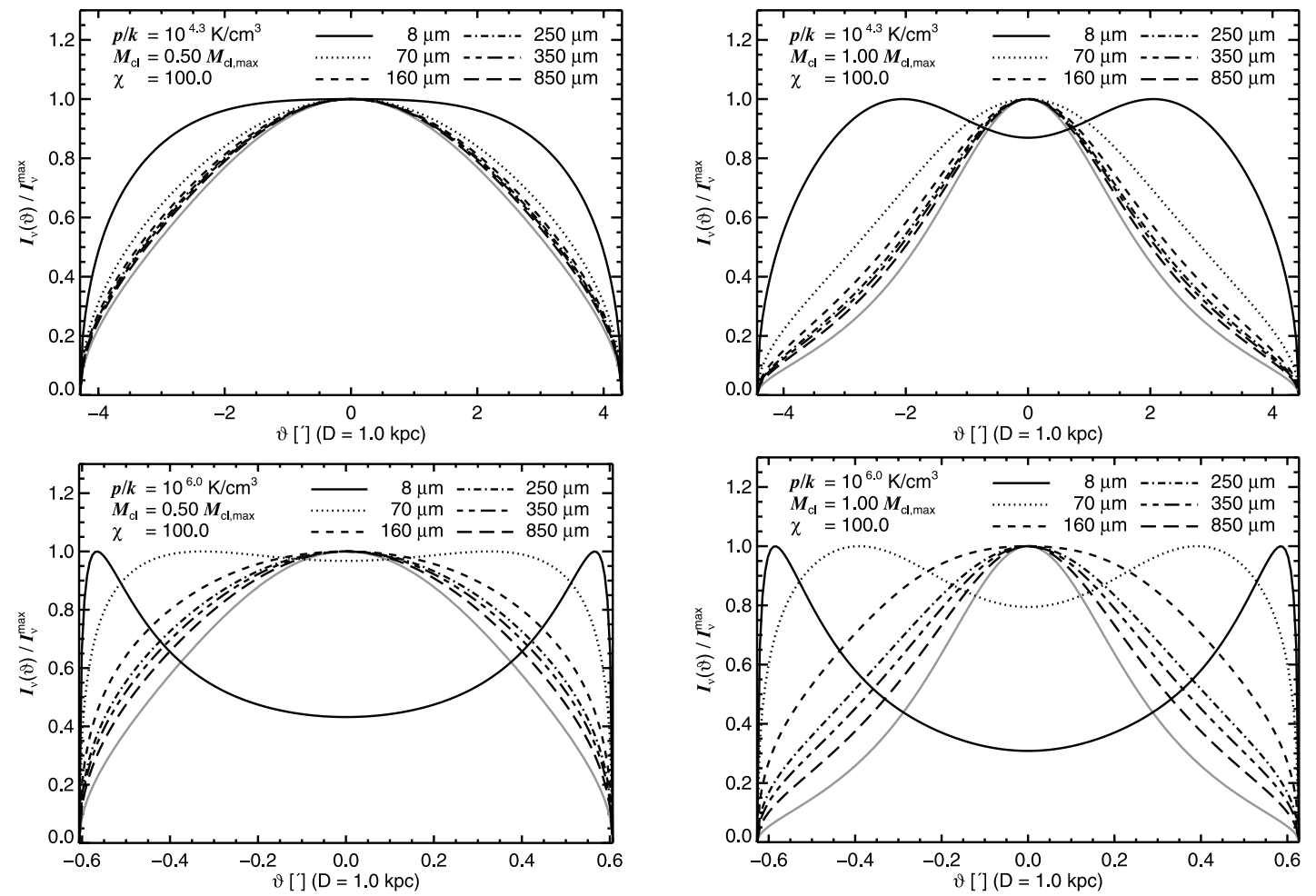

FIG. 14. - Same as Fig. 13, but for a 100 times stronger radiation field. 
TABLE 4

Theoretical Fluxes of the Thermal Emission Spectrum

\begin{tabular}{|c|c|c|c|c|c|c|c|c|}
\hline \multirow[b]{3}{*}{$\begin{array}{c}\lambda_{\text {ref }} \\
(\mu \mathrm{m})\end{array}$} & \multicolumn{4}{|c|}{$p / k=2 \times 10^{4} \mathrm{~K} \mathrm{~cm}^{-3}$} & \multicolumn{4}{|c|}{$p / k=1 \times 10^{6} \mathrm{~K} \mathrm{~cm}^{-3}$} \\
\hline & \multicolumn{2}{|c|}{$f=0.5$} & \multicolumn{2}{|c|}{$f=1.0$} & \multicolumn{2}{|c|}{$f=0.5$} & \multicolumn{2}{|c|}{$f=1.0$} \\
\hline & $\begin{array}{c}\nu I^{(3)}(0)^{\mathrm{a}} \\
\left(10^{-7} \mathrm{~W} \mathrm{~m}^{-2} \mathrm{sr}^{-1}\right)\end{array}$ & $\begin{array}{c}\nu F^{(3)}{ }_{\nu} \\
\left(10^{-7} \mathrm{~W} \mathrm{~m}^{-2}\right)\end{array}$ & $\begin{array}{c}\nu I^{(3)}(0) \\
\left(10^{-7} \mathrm{~W} \mathrm{~m}^{-2} \mathrm{sr}^{-1}\right)\end{array}$ & $\begin{array}{c}\nu F^{(3)}{ }_{\nu} \\
\left(10^{-7} \mathrm{~W} \mathrm{~m}^{-2}\right)\end{array}$ & $\begin{array}{c}\nu I^{(3)}{ }_{\nu}(0) \\
\left(10^{-7} \mathrm{~W} \mathrm{~m}^{-2} \mathrm{sr}^{-1}\right)\end{array}$ & $\begin{array}{c}\nu F^{(3)}{ }_{\nu} \\
\left(10^{-7} \mathrm{~W} \mathrm{~m}^{-2}\right)\end{array}$ & $\begin{array}{c}\nu I^{(3)}(0) \\
\left(10^{-7} \mathrm{~W} \mathrm{~m}^{-2} \mathrm{sr}^{-1}\right)\end{array}$ & $\begin{array}{c}\nu F^{(3)}{ }_{\nu} \\
\left(10^{-7} \mathrm{~W} \mathrm{~m}^{-2}\right)\end{array}$ \\
\hline \multicolumn{9}{|c|}{$\chi=1$} \\
\hline $7.8727^{\mathrm{b}} \ldots \ldots \ldots \ldots \ldots \ldots \ldots \ldots$ & 1.458 & 3.528 & 1.386 & 3.890 & 0.918 & 4.710 & 0.679 & 4.560 \\
\hline $23.843^{\mathrm{b}} \ldots \ldots \ldots \ldots \ldots \ldots \ldots$ & 0.310 & 0.708 & 0.333 & 0.807 & 0.231 & 1.054 & 0.181 & 1.037 \\
\hline $72.556^{\mathrm{b}} \ldots \ldots \ldots \ldots \ldots \ldots \ldots \ldots$ & 1.389 & 2.937 & 1.686 & 3.415 & 1.097 & 4.367 & 0.886 & 4.170 \\
\hline $156.893^{\mathrm{b}} \ldots \ldots \ldots \ldots \ldots \ldots$ & 4.987 & 9.549 & 9.323 & 13.28 & 9.063 & 25.81 & 8.263 & 27.40 \\
\hline $250^{\mathrm{c}}$ & 2.280 & 4.217 & 5.141 & 6.360 & 6.261 & 15.08 & 7.594 & 17.97 \\
\hline $350^{\mathrm{c}}$ & 0.864 & 1.568 & 2.139 & 2.466 & 2.910 & 6.447 & 4.373 & 8.313 \\
\hline $850^{\mathrm{c}}$ & 0.028 & 0.049 & 0.079 & 0.082 & 0.127 & 0.249 & 0.280 & 0.371 \\
\hline \multicolumn{9}{|c|}{$\chi=100$} \\
\hline 7.8727........................ & 146.1 & 353.6 & 138.8 & 389.7 & 91.94 & 471.7 & 68.02 & 456.7 \\
\hline $23.843 \ldots \ldots \ldots \ldots \ldots \ldots \ldots \ldots \ldots$ & 55.17 & 126.3 & 56.86 & 141.1 & 37.09 & 172.9 & 28.28 & 167.3 \\
\hline $72.556 \ldots \ldots \ldots \ldots \ldots \ldots \ldots \ldots$ & 471.6 & 911.3 & 857.6 & 1250 & 815.0 & 2366. & 727.2 & 2489. \\
\hline $156.893 \ldots \ldots \ldots \ldots \ldots \ldots \ldots . .$. & 110.9 & 202.4 & 268.2 & 314.8 & 356.4 & 803.9 & 521.6 & 1021. \\
\hline 250 & 18.54 & 33.13 & 49.77 & 54.03 & 74.82 & 153.9 & 144.0 & 216.7 \\
\hline 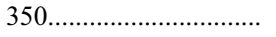 & 4.633 & 8.196 & 13.01 & 13.65 & 20.59 & 40.77 & 44.73 & 60.23 \\
\hline $850 \ldots$ & 0.085 & 0.148 & 0.254 & 0.254 & 0.431 & 0.809 & 1.103 & 1.282 \\
\hline
\end{tabular}

${ }^{a}$ Brightness toward the cloud center.

${ }^{b}$ Reference wavelengths of IRAC 4 and the three MIPS filters of the Spitzer satellite.

${ }^{c}$ Fluxes given for narrowband filter. 
Disk galaxies should be characterized by a radially decreasing global pressure profile. Self-gravitating clouds in low-pressure regions have to be much more massive to become gravitationally unstable. They are less compact, but they are also optically thinner. Therefore, the scale of both the dense clouds and the ISM structure should decrease toward the Galactic center. Equally, stable clouds at larger scale height should be more extended and optically thinner in comparison with clouds located in the center of the thin disk.

\subsection{Effect of the "Clumpy" Medium on the SED}

To analyze the effect of an inhomogeneous ISM on the global SED, we considered an idealized model in which the dust is located in isothermal clouds of the same size and in which the clouds are heated by the same mean radiation field, which is taken to be the ISRF. We assumed that the clouds are embedded in a medium with $p_{\text {ext }} / k=2 \times 10^{4} \mathrm{~K} \mathrm{~cm}^{-3}$ and varied the mass fraction $f$ from $0.1 \%$ up to $100 \%$ of the critical mass. By doing this we covered a range of different optical depths in $V$ toward the cloud center from 0.08 up to 3.57 so that the cloud with the smallest mass fraction can be considered optically thin. We used this spectrum as a reference. The model implies that we have for each cloud size or mass fraction the same total dust mass but only distributed in a different number of clouds.

An interesting question is how much the color $F_{\lambda} / F_{11.3 \mu \mathrm{m}}$ might vary because of the inhomogenous medium, which increases both toward longer wavelength and higher mass fraction. The color $F_{60 \mu \mathrm{m}} / F_{11.3 \mu \mathrm{m}}$ is almost insensitive to the cloud mass, showing a variation of less than $4 \%$. The increase of the color $F_{100 \mu \mathrm{m}} / F_{11.3 \mu \mathrm{m}}$, on the other hand, can be up to $\sim 40 \%$. At even longer wavelengths the difference in color can be larger than a factor of 2 if the clouds are close to gravitational instability.

Since grains in the dense clouds are only heated by a strongly attenuated photon field, the conversion efficiency of the illuminating flux into thermal emission is decreased with respect to the emission of less compact clouds. To obtain an estimate of the decrease of the total emission from the dust clouds, we normalized the total IR emission $L_{\lambda}=4 \pi r_{\mathrm{cl}}^{2} F_{\lambda}$ by the cloud mass $M_{\mathrm{cl}}$. The result is shown in Figure 15.

As can be seen, the "clumpy" density structure leads to a lower total flux in the reemitted spectrum at all wavelengths, an effect that becomes, as expected, stronger with mass fraction. Because of the colder grain temperatures and the resulting shift of the dust emission spectrum toward longer wavelengths, the flux at longer wavelengths are, in general, less affected. We obtain the strongest decrease of the total flux for the PAH emission and the warm dust emission at $60 \mu \mathrm{m}$. Their flux is, at most, $70 \%$ lower compared to a homogeneous medium. At $1000 \mu \mathrm{m}$ the decrease of the total flux is only $\sim 20 \%$.

\subsection{Limitations of the Dust Model}

One main assumption made in this paper to obtain the SED of isothermal clouds is that the grain properties inside the clouds are the same as in the diffuse ISM. In compact clouds it is generally thought that dust growth occurs because of dust accretion and adsorption of atoms and molecules from the gas phase.

Grain growth would have the effect that the extinction curve becomes shallower in the optical and UV/FUV as the smallest grains become less abundant. A measure of the rise of the extinction curve is given by the absolute-to-relative extinction $R_{V}=$ $A_{V} / E(B-V)$, which is higher for flatter curves. Whittet et al. (2001) observed an increase of $R_{V}$ in the Taurus dark clouds for $A_{V}>3$. In addition the $3 \mu \mathrm{m}$ water ice feature started to appear,

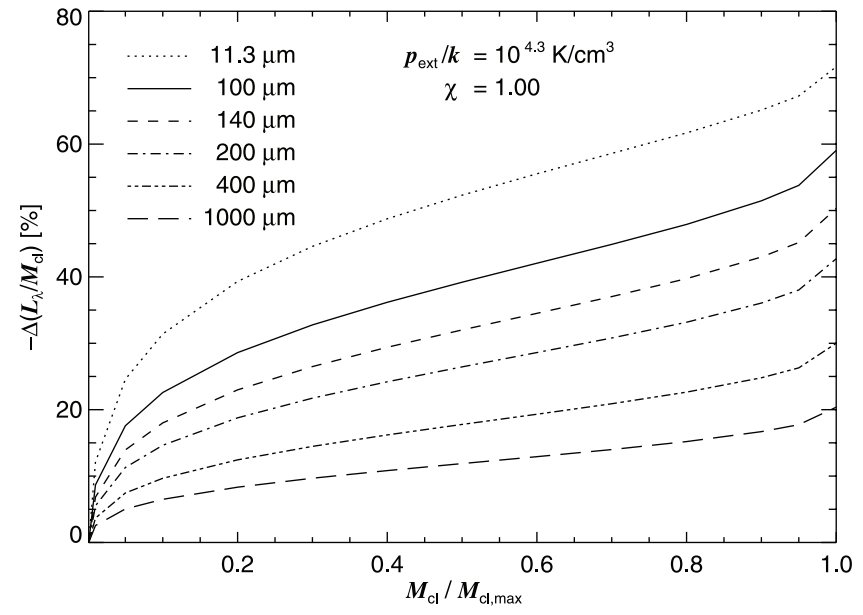

FIG. 15.- Variation of the normalized luminosity $L_{\lambda} / M_{\mathrm{cl}}$ relative to a cloud with $f=0.001$ at several wavelengths as a function of the mass fraction. The outer pressure is taken to be $2 \times 10^{4} \mathrm{~K} \mathrm{~cm}^{-3}$.

in agreement with the expectation of the formation of ice mantles on the grain surfaces in dense gas.

Based on the model of isothermal clouds, we can estimate the corresponding critical density for a change of the grain properties using the central density as the main indicator. In the local diffuse ISM with $p_{\text {ext }} / k \approx 2 \times 10^{4} \mathrm{~K} \mathrm{~cm}^{-3}$ the cloud mass would need to be close to the critical value $(f \gtrsim 0.7)$ to produce a total extinction as large as $A_{V}>3$ (Fig. 4). The kinetic temperatures inside a sample of translucent clouds observed with Far Ultraviolet Spectroscopic Explorer lie in the total range 40-100 K with an average value $68 \pm 15 \mathrm{~K}$ (Rachford et al. 2002). The value is in agreement with the kinetic temperatures of $77 \pm 17 \mathrm{~K}$ derived for 61 sight lines with $\log N_{2}>18.0$ observed with the Copernicus satellite (Savage et al. 1977). Following Rachford et al. the mean kinetic temperature drops to $55 \pm 8 \mathrm{~K}$ after using only a subsample of sight lines with $\log N\left(\mathrm{H}_{2}>20.4\right)$, consistent with their own sample. From Figure 2 we conclude that processes responsible for a flattening of the extinction curve occur at a central density of $n_{\mathrm{H}} \sim 1000 \mathrm{~cm}^{-3}$.

How the grain growth process affects the optical thickness of the cloud is not certain. It is believed that the extinction in the NIR is rather insensitive to the actual grain size distribution. For the effect in the optical one might consider the extinction curve toward $\rho$ Oph A. The curve is not only unusually flat with $R_{V}=$ 4.34 (Cardelli et al. 1989) but also shows with $N_{\mathrm{H}} / E(B-V)=$ $9.3 \times 10^{21} \mathrm{~cm}^{-2} \mathrm{mag}^{-1}$ (Diplas \& Savage 1994) a significantly higher gas-to-dust ratio relative to the mean value of $5.8 \times$ $10^{21} \mathrm{~cm}^{-2} \mathrm{mag}^{-1}$ found for the diffuse ISM (Bohlin et al. 1978), which is also used in this work. If correct and the situation can be generalized, the grain growth process leads to clouds that are optically thinner. The effect should be more dramatic in the UV/ FUV, which is mostly responsible for the grain heating in the diffuse ISM.

Measurements of the extinction curve through translucent clouds furthermore show a broader feature at $2200 \AA$ (Boulanger et al. 1994; Rachford et al. 2002). Boulanger et al. (1994) found for their extinction curves of different sight lines toward the Chameleon cloud complex that the feature becomes not only broader but also weaker if the clouds are optically thicker. This indicates that the carriers inside dense clouds become less abundant, presumably because they stick to larger grains. If our dust model is correct and the $2200 \AA$ feature is related to the PAH molecules, their emission is expected to be weaker. 
Grain growth processes in clouds are also indicated by IR observations (Laureijs et al. 1989; Bernard et al. 1992, 1993; Rawlings et al. 2005). Based on their simplified model of the radial density structure and on the assumption that the clouds are illuminated by an isotropic ISRF, Bernard et al. (1992) concluded that the observed brightness profiles of clouds with a total attenuation as low as 2-4 cannot be purely explained by radiative transfer effects. Bernard et al. (1993) used the dust composition as a free parameter to fit the observed brightness profile of dense clouds. For comparison with observations they also had the cloud illuminated by a nearby young star. Applying this method, they obtained for dense clouds an underabundance of PAHs and very small grains in the central region.

One of their IR sources was the dark cloud G 300.2-16.8, which is thought to be mainly heated by the ISRF (Laureijs et al. 1989; Bernard et al. 1993). Local variations of the dust properties have also been suggested by Rawlings et al. (2005) to explain the strong variations of the IR spectra taken toward three different sight lines (Lemke et al. 1998) that show a change of the PAH emission by a factor of $\sim 2$, which is also much stronger than is predicted by our model calculations. Following Rawlings et al. (2005), the maximum extinction through the cloud is $A_{V} \sim$ 3.5 mag so that a larger grain population in the region of high column density would be in agreement with the findings of Whittet et al. (2001).

The effect of grain growth on the SED should be even more evident in Bok globules, which are characterized by very low temperatures around $10 \mathrm{~K}$. Their densities are therefore above $n_{\mathrm{H}} \sim 10^{3} \mathrm{~cm}^{-3}$, our expected critical value for grain growth in clouds.

Several observations of scattered and emitted light indicate a larger grain population in globules relative to the diffuse ISM (Fitzgerald et al. 1976; Lehtinen \& Mattila 1996; Lehtinen et al. 1998). Fitzgerald et al. (1976) concluded from modeling the scattered optical light from the Thumbprint Nebula that the grain population should produce a rather flat extinction curve with $R_{V}=5.7 \pm 1.1$. This result has been verified by modeling the scattered light in the NIR in $J, H$, and $K$ by Lehtinen \& Mattila (1996). A population of larger grains seems to be supported by observations made at 100 and $200 \mu \mathrm{m}$ (Lehtinen et al. 1998), as the fluxes seen toward the cloud center gave a color temperature significantly smaller than the theoretical predictions presented by Bernard et al. (1992).

\subsubsection{PAH Emission Spectrum}

The strength of the PAH emission features depends on the ionization state of the PAH molecules. The model of Li \& Draine (2001) differentiates between uncharged and charged states. In general the ionization states will vary inside the clouds. In particular, one would expect that the probability of charged PAH molecules decreases inwards with the decrease in the strength of the UV field. Our calculations assume the same probabilities of ionized and nonionized molecules taken to be the values for the CNM. Therefore, the model could be improved by adding an explicit solution of the PAH charge state, and this would then enable the PAH spectrum to be used as a probe of the UV field within the dark cloud.

\subsection{Comparison with Dense Core Models}

The model presented here can be compared with the work of Evans et al. (2001) and of Stamatellos \& Whitworth (2003) who were modeling the emission in the FIR and submillimeter regime of highly dense clouds to obtain better estimates for the dust mass.
As in our model, the calculations were (at least partly) based on an isothermal self-gravitating sphere. However, they applied the model also to unstable clouds, which show higher overpressures and can be optically thicker relative to the critical stable clouds. One of the main differences to our calculations, apart from the fact that Evans et al. (2001) did not consider the complication through scattered light, lies in the method used to derive the thermal emission from dust grains.

In their models, the dust emission at different depths inside the cloud is obtained assuming that the dust emission can be described by an effective grain temperature at which the cooling rate is equal to the heating rate, with both derived using the mean optical dust properties. Although this assumption fulfills energy conservation, it does not necessarily reflect the actual dust temperatures and, therefore, the correct shape of the dust emission spectrum. We have seen that this might only be true in the innermost core region of highly optical thick clouds. Otherwise the simplification will underestimate the dust emission at short wavelengths where it is dominated by the emission from small particles, as they cool, in general, less efficiently and might show strong temperature fluctuations. In the FIR or submillimeter regime a physical model of the grain temperatures might be less important, as the emission is dominated by large grains. However, this aspect should be analyzed more quantitatively to improve the mass estimate for very compact and dense clouds.

The dust composition and the optical properties in the dense medium are major uncertainties. It is possible that these overshadow the uncertainties that arise by using a rather simplified dust model. In both works mentioned above, the calculations are based on the mean dust opacities provided by Ossenkopf \& Henning (1994; OH model), which were derived from theoretical considerations of grain growth in a dense medium and may, therefore, be more appropriate for the dust properties in dense compact clouds. As the data are given only for wavelengths larger then $1 \mu \mathrm{m}$, extrapolations to the optical and UV were made, independent of the actual grain properties assumed by Ossenkopf \& Henning (1994). Stamatellos \& Whitworth (2003) combined the OH model with the model of Mathis et al. (1977; MRN model), which was originally obtained by fitting the mean extinction curve of the ISM. ${ }^{3}$ The MRN model predicts an overabundance of small dust particles to explain the rise in the UV and the optical, while, as discussed in $\S 6.2$, a flat extinction curve is expected. Although it is not mentioned, a flatter curve was probably assumed in the work of Evans et al. (2001), as indicated by the lower effective dust temperature at the outskirts of the clouds (14 K instead of $17 \mathrm{~K}$ as stated by Stamatellos \& Whitworth 2003). For comparison, in our model the equilibrium grain temperatures (shown in Fig. 8) vary between different sizes and compositions. If we consider only the silicate and graphite particles, the temperatures at the edge of highly optical thick clouds range from 12 to $18 \mathrm{~K}$.

The absorption properties in the optical determine not only the effective temperature in the outskirts of the highly optically thick clouds but also how deep the optical emission can penetrate inside the cloud. The stronger temperature gradient in the case of Stamatellos \& Whitworth (2003) should produce a steeper brightness profile at the FIR and submillimeter wavelengths if compared with Evans et al. (2001). It is true that in highly dense clouds most

\footnotetext{
3 However, it is not mentioned why the strong absorption peak at $2200 \AA$ is missing even though the calculations were based on the optical properties of Draine \& Lee (1984), where the bump would be produced by small graphite particles.
} 
of the optical emission is absorbed at the cloud outskirts so that the dust in the core region is predominantly heated by IR photons. However, even if we take into account the DIRBE measurements of the IR emission over the entire sky, which have been neglected in our calculations but have been considered in the two other works, most power of the diffuse ISRF lies in the optical. As a consequence, the emission from the outskirts should affect to a certain degree not only the spectral shape but also, the position of the emission peak. On the other hand, it is indeed expected that the dependence of the SED beyond the emission peak on the optical absorption is not as strong and weakens toward optically thicker clouds.

To study the dependence of the optical properties on the dust emission spectrum, Stamatellos \& Whitworth (2003) considered different scattering properties of the dust grains. The authors found only a weak dependence of $10 \%-20 \%$. The conclusion would be in agreement with our calculations if we had also based them on an effective dust temperature. As we have seen in $\S 5.1$ the dust-scattered light makes only a minor contribution to the dust heating at the cloud outskirts. Where it is important, the heating by scattered light is less than $50 \%$ of the total heating rate. As the effective temperature depends only as $T_{\text {eff }} \propto h(r)^{1 / 6}$ on the heating rate $h(r)$, the effective temperature would not vary by more than a factor of $\sim 2^{-1 / 6}$ if we would have ignored the scattered light completely. Because in the Rayleigh-Jeans part the emission spectrum is proportional to the grain temperature, only a weak temperature dependence beyond the emission peak would arise.

\subsection{The Clouds' Structure}

The shape of the cloud will have certainly an effect on the radiative transfer problem. As mentioned, a future paper will focus on the SED from cylindrical cloud structures where this aspect will, at least to a certain degree, be considered.

Apart from the actual overall shape, the SED of interstellar clouds will be affected by the inhomogeneous substructure caused by nonthermal motion, which is probably related to magnetohydrodynamic turbulence inside the cloud (Curry \& McKee 2000). The turbulent motion will provide an additional pressure-support term, so such clouds might be more stable against gravitational collapse. As discussed for the ISM, the turbulent motion will create a broad density distribution of the local density that is approximately described by a simple lognormal function.

The effect of the turbulent substructure might be considered by using an idealized structure of a turbulent medium superimposed on the simple radial structure discussed here. The turbulent motion might even lead to a broadening so that the radial density profile of turbulent clouds might be shallower. The maximum structure is related to the cloud size, and the width of the density distribution of the local density is related to the strength of the turbulence.

Because of the fractal structure of high-density clouds, the light will penetrate to deeper cloud regions so that grains in those regions become warmer than in clouds without turbulence. In addition turbulence produces density enhancements that are optically thick where grains show colder temperature. It will be important to determine how large these effects actually are.

Our model still does not explain all the observational properties, particularly those of dense clouds. In the case of Bok globules or dense cores in molecular clouds, it has been found that the nonthermal contribution to the line width increases for optically thicker lines, indicating an increase of the turbulent motion from the cloud center outwards (Caselli \& Myers 1995). This observa- tion is generally explained by the strong dissipation of magnetic motion in high-density gas. This leads to a core-envelope structure of the cloud where the core region is mainly supported by thermal pressure while the envelope is supported mainly by magnetic and turbulent pressure. Based on this observation Curry \& McKee (2000) suggested a model in which the core and the envelope are described by two different polytropes, where in the two regions the pressure is related to the density via $p \propto \rho^{\gamma}$ with different values for $\gamma=1+1 / n$, where $n$ is the polytrope index. This model is able to produce for a number of clouds the high density contrast observed, which is larger than the maximum value of 14.04 of the Bonnor-Ebert sphere. McKee \& Hollimann (1999) discussed a model for molecular clouds using multipressure polytropes where the different pressure components in the cloud - thermal, turbulent, and magnetic pressure-are described by different polytropes. It produces arbitrarily large density contrasts for stable clouds.

On the other hand, it has been shown based on extinction measurements in the NIR that the profiles of the projected column density of rather spherical globules are well described by the density structure of the isothermal sphere (Alves et al. 2001; Kandori et al. 2005). The small sample of 10 globules studied by Kandori et al. indicate that most of the globules are critical to gravitational collapse. The derived external pressure, however, shows strong variation from $2.1 \times 10^{4}$ up to $1.8 \times 10^{5} \mathrm{~K} \mathrm{~cm}^{-3}$, with a mean of $5.7 \times 10^{4} \mathrm{~K} \mathrm{~cm}^{-3}$, which is almost 3 times larger than the value assumed here, which should be typical for the ISM of our Galaxy. It might well be that in certain cases the simplification using an isothermal cloud model has to be compensated by a higher external pressure. Following Kandori et al., the model of an isothermal sphere is also able to mimic in the limit of the observational uncertainties the density profiles of clouds that undergo gravitational collapse.

\section{SUMMARY}

We have analyzed in some detail the SED from spherical, selfgravitating, isothermal stable clouds illuminated by an isotropic ISRF. The radiative transfer through those clouds is, for a given radiation field and dust properties inside the cloud, determined by the pressure of the ambient medium and by the critical mass fraction $f=M_{\mathrm{cl}} / M_{\mathrm{cl} \text {, max }}$ of the cloud mass $M_{\mathrm{cl}}$ and the critical mass $M_{\mathrm{cl} \text {, max }}$ for which the cloud becomes gravitationally unstable. The dust properties in the clouds are assumed to be consistent with those in the diffuse ISM of our Galaxy. We derived the SED for different assumptions of the critical mass fraction $f$, outer pressure $p_{\text {ext }}$, and strength of the external ISRF. Special attention was paid to the SED from clouds embedded in the WNM with a pressure of $p_{\text {ext }} / k=2 \times 10^{4} \mathrm{~K} \mathrm{~cm}^{-3}$, which were compared with SEDs from clouds in higher pressure regions. We find that

1. The grain temperatures in stable isothermal clouds embedded in the WNM are generally higher than $10 \mathrm{~K}$. Lower temperatures are expected for collapsing clouds or for clouds in higher pressure regions.

2. The ratio of the dust emission relative to the PAH emission rises toward higher critical mass fractions and outer pressures.

3. As clouds are generally optically thicker in higher pressure regions, more light is transferred to thermal emission.

We also analyzed the brightness profiles of the scattered emission at 440 and at $900 \mathrm{~nm}$ and the thermal emission from dust grains and PAH molecules in the IR/FIR and in the submillimeter/ millimeter regime. We find that 
1. For stable isothermal clouds embedded in the WNM, the scattered emission at $440 \mathrm{~nm}$ develops for increasing mass fraction a broad winglike profile as seen for Bok globules in our Galaxy. At longer wavelengths the scattered emission is brighter toward the cloud center. In high-pressure regions the scattered emission is concentrated at the limb of the clouds.

2. For stable isothermal clouds in the WNM the PAH brightness profile shows a weak winglike profile if the clouds are critical to collapse. In high-density regions the profile shows a strong limb-brightening effect.

3. The brightness profiles of the dust emission is generally broader with respect to the profile of the column density. The effect increases toward shorter wavelengths and is more prominent in high-pressure regions.

M. A. D. acknowledges the support of both the Australian National University and of the Australian Research Council through his ARC Australian Federation Fellowship. Both authors acknowledge financial support for this research through an ARC Discovery project grant DP0208445. J. F. acknowledges helpful discussions he had with Ute Liesenfeld, Endrik Krügel, and Richard Tuffs.

\section{APPENDIX}

\section{THE PHYSICAL PARAMETERS OF ISOTHERMAL CLOUDS}

Here we want to justify our statements that both the density profile and (for a given outer pressure) the column density to the center of gravitationally stable isothermal clouds can be characterized by the critical mass fraction $M_{\mathrm{cl}} / M_{\mathrm{cl}}$, max , where $M_{\mathrm{cl}}$ is the cloud mass and $M_{\mathrm{cl} \text {, max }}$ is the maximum cloud mass critically stable against gravitational collapse. In addition we provide the accurate expression for the cloud size.

The Ansatz of self-gravitating isothermal spherical clouds leads to the famous Lane-Emden equation, a differential equation of the unit-free potential $\omega=\Phi / K$, where $\Phi$ is the gravitational potential and $K=k T_{\mathrm{cl}} / \mu m_{\mathrm{u}}$. Here, $k, T_{\mathrm{cl}}, \mu$, and $m_{\mathrm{u}}$ are the Boltzmann constant, the cloud temperature, the mean molecular weight, and the atomic mass unit, respectively. The Lane-Emden equation is given by

$$
\frac{d^{2} \omega}{d z^{2}}+\frac{2}{z} \frac{d \omega}{d z}=e^{-\omega}
$$

where $z=A r$, with $r$ as the radius and $A^{2}=4 \pi G \rho_{c} / K$. The density in the cloud center is $\rho_{c}$, and the gravitational constant is $G$. The radial density and the pressure, respectively, are simply given by the exponentials:

$$
\rho(z)=\rho_{c} e^{-\omega(z)}, \quad p(z)=p_{c} e^{-\omega(z)}
$$

where $p_{c}=K \rho_{c}$.

First we consider the pressure at the outer radius of a cloud of certain mass $M_{\mathrm{cl}}$. We express the constant $A$ through the mass $M_{\mathrm{cl}}$ of the cloud:

$$
A\left(z_{\mathrm{cl}}\right)=\frac{K}{M_{\mathrm{cl}} G} \int_{0}^{z_{\mathrm{cl}}} d z^{\prime} z^{\prime 2} e^{-\omega\left(z^{\prime}\right)}
$$

If we replace $\rho_{c}$ by $A^{2} K /(4 \pi G)$ and the constant $A$ through equation (A3), we obtain for the pressure of a cloud with mass $M_{\mathrm{cl}}$ the following variation as a function of the parameter $z_{\mathrm{cl}}$ :

$$
p\left(z_{\mathrm{cl}}\right)=\frac{K^{4}}{4 \pi G^{3} M_{\mathrm{cl}}^{2}} e^{-\omega\left(z_{\mathrm{cl}}\right)}\left(\int_{0}^{z_{\mathrm{cl}}} d z^{\prime} z^{\prime 2} e^{-\omega\left(z^{\prime}\right)}\right)^{2} .
$$

The variation of the outer pressure as a function of $z_{\mathrm{cl}}$ is shown in Figure 16 (right). For small values the pressure increases as a function of $z_{\mathrm{cl}}$ up to a maximum at $z_{\max } \approx 6.451$. At $z_{\mathrm{cl}}>z_{\max }$ the function shows smooth variations with declining amplitude around a certain pressure value. The solutions for the isothermal clouds that are stable against gravitational collapse lie in the region $z_{\mathrm{cl}} \leq z_{\mathrm{max}}$. The maximum pressure is related to a maximum stable cloud mass $M_{\mathrm{cl} \text {, max }}$ :

$$
M_{\mathrm{cl}, \max }\left(z_{\max }\right)=\frac{K^{2}}{\sqrt{4 \pi G^{3} p_{\mathrm{ext}}}} e^{-\omega\left(z_{\max }\right) / 2} \int_{0}^{z_{\max }} d z^{\prime} z^{\prime 2} e^{-\omega\left(z^{\prime}\right)} .
$$

In this paper we have chosen to measure the cloud mass $M_{\mathrm{cl}}$ in units of the critical mass of a cloud with the same temperature. If we replace the cloud mass by $f=M_{\mathrm{cl}} / M_{\mathrm{cl} \text {, max }}$, the pressure at the outer cloud radius is given by

$$
\frac{p\left(z_{\mathrm{cl}}\right)}{p_{\mathrm{ext}}}=\frac{1}{f^{2}} \frac{e^{-\omega\left(z_{\mathrm{cl}}\right)}}{e^{-\omega\left(z_{\max }\right)}}\left[\frac{\int_{0}^{z_{\mathrm{cl}}} d z z^{2} e^{-\omega(z)}}{\int_{0}^{z_{\max }} d z z^{2} e^{-\omega(z)}}\right]^{2} .
$$



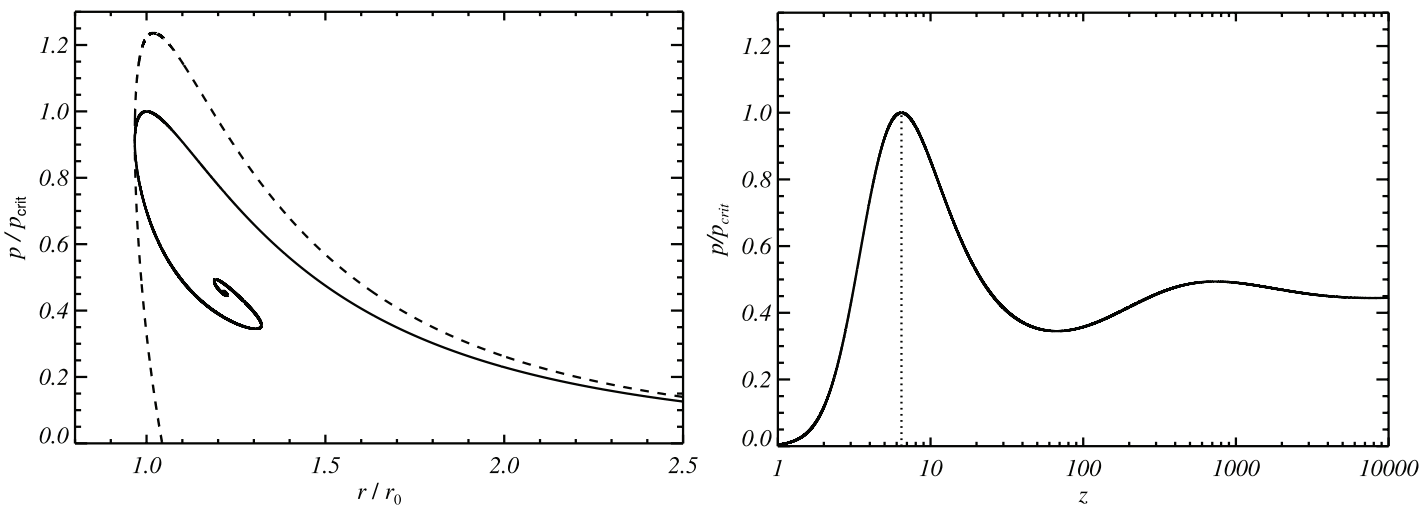

FIG. 16.- Normalized pressure $p / p_{\text {crit }}$ at the outer boundary of the isothermal sphere as a function of the normalized radius $r / r_{0}$ where $r_{0}=0.411 M_{\mathrm{cl}} G / K(l e f t)$ and as a function of $z($ right $)$. The quantity $G$ is the gravitational constant and $K=k T_{\mathrm{cl}} /\left(\mu m_{\mathrm{u}}\right)$, where $m_{\mathrm{u}}$ and $\mu$ are the atomic mass unit and the mean molecular weight, respectively. The dashed line in the left panel is derived using a simple application of the virial theorem, while the solid curve is obtained by solving the Lane-Emden equation. The dotted curve in the right panel marks the boundary where the clouds become gravitationally unstable, which defines $z_{\max }$. The solutions considered in this paper of gravitational stable clouds lie to the left.

In the region of stable clouds that are in pressure equilibrium with their external medium $\left[p_{\mathrm{ext}}=p\left(z_{\mathrm{cl}}\right)\right]$ the value $z_{\mathrm{cl}}$ can be considered as a pure function of $f$.

Often the model is also applied to clouds that are in a state of collapse or show, in general, a higher overpressure than for critical stable clouds. In this situation it is not clear how well the pressure $p\left(z_{\mathrm{cl}}\right)$ at the cloud edge reflects the external pressure $p_{\text {ext }}$. From Figure 16 we see that the pressure at the outskirts would drop below the external pressure if the cloud becomes unstable. However, the isothermal cloud model seems to be useful as an approximate solution of the density structure of unstable clouds as it resembles the power-law density profile close to $\rho(r) \propto r^{-2}$ at the outskirts and a flat profile in the center as observed for prestellar cores.

The radial density and pressure profile is directly obtained by the solution of the Lane-Emden equation:

$$
\frac{\rho(z)}{\rho\left(z_{\mathrm{cl}}\right)}=\frac{p(z)}{p\left(z_{\mathrm{cl}}\right)}=\frac{e^{-\omega(z)}}{e^{-\omega\left(z_{\mathrm{cl}}\right)}} .
$$

Because the transformation from $z$ to $r$ is linear, the same applies to the density profile $\rho(r) / \rho\left(r_{\mathrm{cl}}\right)=p(r) / p\left(r_{\mathrm{cl}}\right)$. As $\omega(z=0)=0$, the central density is given by

$$
\rho_{c}=\frac{p\left(z_{\mathrm{cl}}\right) e^{\omega\left(z_{\mathrm{cl}}\right)}}{K}
$$

An equally simple relation can be found for the cloud radius:

$$
r_{\mathrm{cl}}=z \frac{K}{\sqrt{4 \pi G p\left(z_{\mathrm{cl}}\right)}} e^{-\omega\left(z_{\mathrm{cl}}\right) / 2} .
$$

The last parameter of the isothermal clouds we consider is the column density, given by

$$
N_{\mathrm{H}}=\int d r n_{\mathrm{H}}(r)=\left(m_{\mathrm{u}} \sum_{i} \frac{n_{i}}{n_{\mathrm{H}}} \mu_{i}\right)^{-1} \int_{0}^{r_{\mathrm{cl}}} d r \rho(r),
$$

where $n_{i}$ is the number density of element $i$ and $\mu_{i}$ is its mean molecular weight. Using the solution of the isothermal cloud for its radius, density, and pressure, one finds

$$
N_{\mathrm{H}}\left(z_{\mathrm{cl}}\right)=\left(m_{\mathrm{u}} \sum_{i} \frac{n_{i}}{n_{\mathrm{H}}} \mu_{i}\right)^{-1} \sqrt{\frac{p\left(z_{\mathrm{cl}}\right)}{4 \pi G}} \int_{0}^{z_{\mathrm{cl}}} d z e^{-\omega(z)} e^{\omega\left(z_{\mathrm{cl}}\right) / 2}
$$

For a given parameter $z_{\mathrm{cl}}$ the column density is proportional to the pressure $\left[p\left(z_{\mathrm{cl}}\right)\right]^{1 / 2}$ at the edge of the cloud. 
Alves, J. F., Lada, C. J., \& Lada, E. A. 2001, Nature, 409, 159

Arendt, R. G., et al. 1998, ApJ, 508, 74

Arquilla, R., \& Goldsmith, P. F. 1985, ApJ, 297, 436

Bate, M. R., Bonnell, I. A., \& Bromm, V. 2003, MNRAS, 339, 577

Bernard, J. P., Boulanger, F., Desert, F. X., \& Puget, J. L. 1992, A\&A, 263, 258

Bernard, J. P., Boulanger, F., \& Puget, J. L. 1993, A\&A, 277, 609

Bohlin, R. C., Savage, B. D., \& Drake, J. F. 1978, ApJ, 224, 132

Bok, B. J., \& Reilly, E. F. 1947, ApJ, 105, 255

Bonnor, W. B. 1956, MNRAS, 116, 351

Boulanger, F., Prévot, M. L., \& Gry, C. 1994, A\&A, 284, 956

Boulares, A., \& Cox, D. P. 1990, ApJ, 365, 544

Cardelli, J. A., Clayton, G. C., \& Mathis, J. S. 1989, ApJ, 345, 245

Cardelli, J. A., \& Savage, B. D. 1988, ApJ, 325, 864

Caselli, P., \& Myers, P. C. 1995, ApJ, 446, 665

Chlewicki, G., \& Laureijs, R. J. 1988, A\&A, 207, L11

Curry, C. L., \& McKee, C. F. 2000, ApJ, 528, 734

Dickmann, R. L., \& Clemens, D. P. 1983, ApJ, 271, 143

Diplas, A., \& Savage, B. D. 1994, ApJS, 93, 211

Dopita, M. A., \& Sutherland, R. S. 2003, Astrophysics of the Diffuse Universe (Berlin: Springer)

Dopita, M. A., et al. 2005, ApJ, 619, 755

Draine, B. T., \& Lee, H. M. 1984, ApJ, 285, 89

Draine, B. T., \& Li, A. 2007, ApJ, 657, 810

Ebert, R. 1955, Z. Astrophys., 37, 217

Elmegreen, B. G. 2002, ApJ, 564, 773

Evans, N. J., II, Rawlings, J. M. C., Shirley, Y. L., \& Mundy, L. G. 2001, ApJ, 557, 193

Ferrière, K. M. 2001, Rev. Med. Phys, 73, 1031

Fischera, J. 2000, Ph.D. thesis, Univ. Heidelberg 2004, A\&A, 428, 99

Fitzgerald, M. P., Stephens, T. C., \& Witt, A. N. 1976, ApJ, 208, 709

Fitzpatrick, E. L. 1999, PASP, 111, 63

Gail, H.-P., \& Sedlmayr, E. 1999, A\&A, 347, 594

Guhathakurta, P., \& Draine, B. T. 1989, ApJ, 345, 230

Henyey, L. G., \& Greenstein, J. L. 1941, ApJ, 93, 70

Holweger, H. 2001, in AIP Conf. Proc 598, Solar and Galactic Composition,

ed. R. F. Wimmer-Schweingruber (New York: AIP), 23

Kandori, R., et al. 2005, AJ, 130, 2166

\section{REFERENCES}

Kimura, H., Mann, I., \& Jessberger, E. K. 2003, ApJ, 582, 846

Kippenhahn, R., \& Weigert, A. 1990, Stellar Structure and Evolution (Berlin: Springer)

Krumhansl, J., \& Brooks, H. 1953, J. Chem. Phys., 21, 1663

Laor, A., \& Draine, B. T. 1993, ApJ, 402, 441

Laureijs, R. J., Chlewicki, G., Clark, F. O., \& Wesselius, P. R. 1989, A\&A, 220, 226

Lehtinen, K., Lemke, D., Mattila, K., \& Haikala, L. K. 1998, A\&A, 333, 702

Lehtinen, K., \& Mattila, K. 1996, A\&A, 309, 570

Lehtinen, K., Mattila, K., Schnur, G. F. O., \& Prusti, T. 1995, A\&A, 295, 487

Lemke, D., Mattila, K., Lehtinen, K., Laureijs, R. J., Liljestrom, T., Léger, A., \& Herbstmeier, U. 1998, A\&A, 331, 742

Li, A., \& Draine, B. T. 2001, ApJ, 554, 778

Martin, P. G., \& Whittet, D. G. B. 1990, ApJ, 357, 113

Mathis, J. S., Mezger, P. G., \& Panagia, N. 1982, A\&A, 105, 372

Mathis, J. S., Rumpl, W., \& Nordsieck, K. H. 1977, ApJ, 217, 425

Mattila, K. 1970, A\&A, 9, 53

McKee, C. F., \& Hollimann, J. H., II. 1999, ApJ, 522, 313

Nordlund, Å., \& Padoan, P. 1999, in Interstellar Turbulence, ed. J. Franco \& A. Carramiñana (Cambridge: Cambridge Univ. Press), 218

Ossenkopf, V., \& Henning, T. 1994, A\&A, 291, 943

Padoan, P., \& Nordlund, A. 2002, ApJ, 576, 870

Padoan, P., Nordlund, Å., \& Jones, B. J. T. 1997, MNRAS, 288, 145

Passot, T., \& Vázquez-Semadeni, E., 1998, Phys. Rev. E, 58, 4501

Rachford, B. L., et al. 2002, ApJ, 577, 221

Rawlings, M. G., Juvela, M., Mattila, K., Lehtinen, K., \& Lemke, D. 2005 , MNRAS, 356, 810

Savage, B. D., Bohlin, R. C., Drake, J. F., \& Budich, W. 1977, ApJ, 216, 291 Stamatellos, D., \& Whitworth, A. P. 2003, A\&A, 407, 941

van Dishoeck, E. F., \& Black, J. H. 1988, ApJ, 334, 771

Vázquez-Semadeni, E. 1994, ApJ, 423, 681

Voit, G. M. 1991, ApJ, 379, 122

Weingartner, J. C., \& Draine, B. T. 2001, ApJ, 548, 296

Whittet, D. C. B., Gerakins, P. A., Hough, J. H., \& Shenoy, S. S. 2001, ApJ, 547,872

Witt, A. N., Oliveri, M. V., \& Schild, R. E. 1990, AJ, 99, 888

Yun, J. L., \& Clemens, D. P. 1990, ApJ, 365, L73

Zubko, V., Dwek, E., \& Arendt, R. G. 2004, ApJS, 152, 211 